OPEN ACCESS

Edited by:

Junji Yodoi,

Kyoto University, Japan

Reviewed by: Arvand Haschemi, Medical University of Vienna, Austria Guillaume Tabouret,

National Institute of

Agricultural Research, France

*Correspondence:

Robert J. Wilkinson

r.j.wilkinson@imperial.ac.uk;

Bruno B. Andrade

bruno.andrade@bahia.fiocruz.br

t.:These authors have contributed equally to this work.

Specialty section:

This article was submitted to Inflammation,

a section of the journal

Frontiers in Immunology

Received: 22 February 2017 Accepted: 24 April 2017

Published: 12 May 2017

Citation:

Rockwood N, Costa DL, Amaral EP, Du Bruyn E, Kubler A, Gil-Santana L, Fukutani KF, Scanga CA, Flynn JL, Jackson $S H$, Wilkinson $K A$, Bishai WR, Sher A, Wilkinson RJ and Andrade BB (2017) Mycobacterium tuberculosis Induction of Heme

Oxygenase-1 Expression is

Dependent on Oxidative Stress and Reflects Treatment Outcomes.

Front. Immunol. 8:542. doi: 10.3389/fimmu.2017.00542

\section{Mycobacterium tuberculosis Induction of Heme Oxygenase-1 Expression Is Dependent on Oxidative Stress and Reflects Treatment Outcomes}

\author{
Neesha Rockwood ${ }^{1,2+}$, Diego L. Costa ${ }^{3 \dagger}$, Eduardo P. Amaral ${ }^{3+}$, Elsa Du Bruyn', \\ Andre Kubler 4,5 , Leonardo Gil-Santana ${ }^{6,7,8}$, Kiyoshi F. Fukutani ${ }^{6}$, Charles A. Scanga ${ }^{9}$, \\ JoAnne L. Flynn ${ }^{9}$, Sharon H. Jackson ${ }^{10}$, Katalin A. Wilkinson ${ }^{1,11}$, William R. Bishai ${ }^{5}$, \\ Alan Sher ${ }^{3 \dagger}$, Robert J. Wilkinson ${ }^{1,2,11^{*}}$ and Bruno B. Andrade ${ }^{1,6,7,8,12,13 *}$ \\ ${ }^{1}$ Wellcome Centre for Infectious Disease Research in Africa, Institute of Infectious Disease and Molecular Medicine, \\ University of Cape Town, Cape Town, South Africa, ${ }^{2}$ Department of Medicine, Imperial College, London, UK, \\ ${ }^{3}$ Immunobiology Section, Laboratory of Parasitic Diseases, National Institutes of Allergy and Infectious Diseases, National \\ Institutes of Health, Bethesda, MD, USA, ${ }^{4}$ Infectious Diseases and Immunity, Imperial College, London, UK, ${ }^{5}$ Center for \\ Tuberculosis Research, Johns Hopkins University School of Medicine, Baltimore, MD, USA, ${ }^{6}$ Instituto Gonçalo Moniz, \\ Fundação Oswaldo Cruz, Salvador, Brazil, ' Multinational Organization Network Sponsoring Translational and Epidemiological \\ Research (MONSTER) Initiative, Fundação José Silveira, Salvador, Brazil, ${ }^{8}$ Curso de Medicina, Faculdade de Tecnologia e \\ Ciências, Salvador, Brazil, ${ }^{9}$ Department of Microbiology and Molecular Genetics, University of Pittsburgh School of Medicine, \\ Pittsburgh, PA, USA, ${ }^{10}$ Division of Intramural Research, National Institute on Minority Health and Health Disparities, National \\ Institutes of Health, Bethesda, MD, USA, ${ }^{11}$ The Francis Crick Institute, London, UK, ${ }^{12}$ Division of Infectious Diseases, \\ Department of Medicine, Vanderbilt University School of Medicine, Nashville, TN, USA, ${ }^{13}$ Escola Bahiana de Medicina e \\ Saúde Pública, Salvador, Brazil
}

The antioxidant enzyme heme oxygenase-1 $(\mathrm{HO}-1)$ is implicated in the pathogenesis of tuberculosis (TB) and has been proposed as a biomarker of active disease. Nevertheless, the mechanisms by which Mycobacterium tuberculosis (Mtb) induces $\mathrm{HO}-1$ as well as how its expression is affected by HIV-1 coinfection and successful antitubercular therapy (ATT) are poorly understood. We found that $\mathrm{HO}-1$ expression is markedly increased in rabbits, mice, and non-human primates during experimental Mtb infection and gradually decreased during ATT. In addition, we examined circulating concentrations of $\mathrm{HO}-1$ in a cohort of $130 \mathrm{HIV}-1$ coinfected and uninfected pulmonary TB patients undergoing ATT to investigate changes in expression of this biomarker in relation to HIV-1 status, radiological disease severity, and treatment outcome. We found that plasma levels of HO-1 were elevated in untreated HIV-1 coinfected TB patients and correlated positively with HIV-1 viral load and negatively with $\mathrm{CD} 4^{+} \mathrm{T}$ cell count. In both HIV-1 coinfected and Mtb monoinfected patients, HO-1 levels were substantially reduced during successful TB treatment but not in those who experienced treatment failure or subsequently relapsed. To further delineate the molecular mechanisms involved in induction of $\mathrm{HO}-1$ by $\mathrm{Mtb}$, we performed a series of in vitro experiments using mouse and human macrophages. We found that $M$ tb-induced $\mathrm{HO}-1$ expression requires NADPH oxidase-dependent reactive oxygen species production induced by the early-secreted antigen ESAT-6, which in turn triggers nuclear translocation of the 
transcription factor NRF-2. These observations provide further insight into the utility of $\mathrm{HO}-1$ as a biomarker of both disease and successful therapy in TB monoinfected and HIV-TB coinfected patients and reveal a previously undocumented pathway linking expression of the enzyme with oxidative stress.

Keywords: tuberculosis, HIV, heme oxygenase-1, biomarker, oxidative stress

\section{INTRODUCTION}

Tuberculosis (TB) is an airborne disease caused by infection with Mycobacterium tuberculosis (Mtb) bacilli. It remains a major global health problem as approximately 1.7 billion people are estimated to be infected (1). In 2015, it was estimated that 1.4 million people died from TB alone with an additional 0.4 million deaths as a result of TB-HIV coinfection, making TB 1 of the 10 top causes of morbidity and death worldwide (2).

The pathogenesis of TB involves activation of myeloid cells, which promotes the generation of free radicals $(3,4)$. However, due to the broad diversity of clinical manifestations, TB infection outcomes can vary from asymptomatic infection [latent TB infection (LTBI)] to severe progressive lung disease with extensive destruction of pulmonary tissue (5-7). Both clinical and experimental animal studies in TB have described excessive oxidative cellular and tissue damage leading to lipid peroxidation and cell death (8-11). The intense inflammatory environment of active TB is hallmarked by accumulation of reactive oxygen species (ROS), which are largely driven by mitochondria via NADPH oxidase (12).

Heme oxygenase-1 (HO-1) is a potent antioxidant enzyme, which is induced in response to cellular stress $(10,13,14)$. In several experimental systems, HO-1 expression is triggered in response to heme accumulation (15), exposure to toxic arsenic compounds $(16,17)$, hypoxia (18), starvation (19), as well as tolllike receptor (TLR) and cytokine-mediated cellular activation (20-22). The enzyme catalyzes the degradation of heme molecules into free iron, biliverdin, and carbon monoxide (CO), the latter product exhibiting anti-inflammatory and cytoprotective effects (14). The protective versus detrimental roles of host HO-1 during TB infection are still controversial and incompletely understood. Mice genetically deficient in HO- 1 are more susceptible to mycobacterial infection (23). However, these knockout animals also exhibit profound hematopoietic abnormalities $(24,25)$ that could provide an alternative explanation of the phenotype observed in $M t b$ infection.

There is additional contrary evidence that HO-1 expression may be associated with rather than suppress TB infection and pathology. Indeed, in studies on South Indian and Brazilian patients, circulating levels of $\mathrm{HO}-1$ were increased in both adult and pediatric TB patients compared to uninfected persons and patients with LTBI (26-28). In addition, TB patients exhibiting clinical and radiographic signs of more severe illness displayed significantly higher HO-1 levels in plasma than those who had milder TB disease $(10,27)$. The concept that HO-1 may actually promote $\mathrm{TB}$ infection is supported by experiments in which growth of $M t b$ in macrophages was suppressed by addition of a pharmacological inhibitor of host HO-1 enzymatic activity (29). The same drug when given to $M t b$-infected mice induced a substantial decrease in pulmonary mycobacterial loads when administered in conjunction with anti-TB antibiotic therapy and accelerated bacterial clearance (30). Together, these results argue that in the context of $M t b$ infection, overexpression of HO-1 may be detrimental rather than beneficial.

The molecular mechanisms underlying $\mathrm{HO}-1$ induction by $M t b$ are only partially understood. As mentioned earlier, infection of murine and human macrophages with $M t b$ induces robust production of HO-1 (10) and mycobacterial infection of mice also triggers pulmonary expression of the enzyme (23, 30-32). We have previously shown that HO-1 induction in human macrophages requires live replicating $M t b$ and the expression of ESAT-6 (6 kDa early secretory antigenic target) (10). Nevertheless, the downstream intermediates through which this $M t b$ virulence factor triggers $\mathrm{HO}-1$ transcription have not been defined.

In this study, we have extended our analysis of HO- 1 as a biomarker of active TB infection and further delineated the mechanisms through which $M t b$ induces $\mathrm{HO}-1$ production in infected cells. First, we analyzed in vivo HO-1 expression in experimental $M t b$ infection of rabbits, mice, and non-human primates (NHP) and confirmed in these different animal models that HO-1 levels are associated with active infection and disease and diminish upon antitubercular therapy (ATT). In addition, using plasma samples from a large cohort of clinically well-characterized $\mathrm{TB}$ and HIV-1-TB patients in South Africa, we analyzed for the first time the influence of concomitant HIV-1 infection on TB-induced HO-1 expression and correlated enzyme levels with treatment outcome. Finally, in in vitro studies using infected monocytederived human macrophages and bone marrow-derived murine macrophages, we demonstrated that the mechanism by which Mtb ESAT-6 stimulates HO-1 expression involves the induction of NADPH-derived ROS production leading to activation and translocation of the transcription factor nuclear factor erythroidderived 2-like 2 (NRF-2). Taken together, these findings further delineate the pathway through which $M t b$ infection triggers HO-1 expression while demonstrating the potential use of the enzyme as biomarker for disease and treatment outcome in the complex setting of a population where TB and HIV-1 infections coexist.

\section{RESULTS}

\section{Mtb Infection Drives HO-1 Expression at the Disease Site and Systemically in Three Experimental Models of Pulmonary TB}

We have previously shown that circulating levels of HO-1 are increased in pulmonary $\mathrm{TB}$ patients compared to uninfected 
individuals and those with LTBI in both adults and children $(27,28)$. In these studies, patients were recruited at the time of disease diagnosis and for this reason we did not have data on their history of TB infection. In order to temporally examine the induction of HO-1 following $M t b$ infection, we quantified its expression in three distinct animal models. Using a recently published model of lung cavitation in rabbits $(33,34)$, we observed that HO-1 protein expression increased in lung areas with granulomatous lesions compared to those with normal tissue (Figure 1A). HO-1 expression was further augmented in the cavity walls (Figure 1A). Notably, the level of HO-1 expression reflected bacterial burden in the different lung areas in infected animals (Figure 1A). Pulmonary infection of rabbits with $M t b$ was also associated with a significant increase in HO-1 plasma levels (Figure 1A). These results indicate that circulating levels of $\mathrm{HO}-1$ reflect the induction of HO- 1 protein expression at the infection site. In addition, they show that HO-1 expression correlates with the bacterial burden in different areas of the infected lung.

We next examined HO-1 expression in lungs and in serum in a mouse model of pulmonary TB (35), before and after a chemotherapy regimen that closely mimics that used in humans. In agreement with a recent study from our group (30), we found that HO-1 expression in both lungs and serum significantly increased following aerosol infection with $M t b$ (Figure 1B). Initiation of ATT reduced HO-1 induction, with concentrations substantially falling in the serum of mice receiving the antimicrobials compared to untreated animals (Figure 1B). Interestingly, although greatly reduced, significant levels of HO-1 expression remained relative to the levels displayed by uninfected control mice perhaps due to the presence of persistent bacteria.

Experimental infection of NHP has been described to induce a pulmonary disease that more accurately resembles human $\mathrm{TB}$ than that occurring in murine and rabbit models (36). We therefore measured HO-1 levels in plasma of cynomolgus macaques bronchoscopically infected with $M t b$ and treated with rifampin or isoniazid when active TB disease was established (37). In this NHP model, HO-1 levels significantly increased as early as 4 weeks postinfection and remained elevated following development of active TB (Figure 1C). Treatment with either rifampin or isoniazid was associated with gradual decreases in HO-1 concentrations (Figure 1C). At the end of therapy, treated animals exhibited HO-1 levels indistinguishable from those detected at preinfection. These findings in multiple animal models strongly support the association of HO-1 expression levels with TB infection and disease as well as the potential utility of the enzyme as biomarker of successful anti-TB therapy.

\section{Plasma Levels of HO-1 Distinguish Successful from Failed Treatment or Relapse in Pulmonary TB Patients from South Africa}

Our previous clinical data suggested that measuring HO-1 expression in patients undergoing ATT could potentially discriminate different treatment outcomes (27). To more rigorously test this hypothesis, we examined HO-1 concentrations in plasma from a well-characterized cohort of 130 South African patients with active pulmonary TB from whom serial samples were collected. HO-1 concentrations gradually diminished during treatment (Figure 2A). Notably, while levels of the enzyme consistently decreased in individuals who were successfully treated, they did not significantly change in patients who exhibited unfavorable outcomes (e.g., treatment failure or TB relapse at the end of therapy) (Figure 2B). Plasma concentrations of HO-1 were similar between patients who exhibited TB relapse or treatment failure during the study follow-up irrespective of the time point (Figure 2C), although the small number of patients in these subgroups of unfavorable outcomes preclude a definite conclusion. Similarly, prior to therapy, HO-1 levels were indistinguishable between patients who were successfully treated and those who were not (Figure 2B). Nevertheless, by week 20 of therapy, patients with unfavorable outcomes exhibited HO-1 levels substantially higher than those successfully treated (Figure 2B). These findings demonstrate that pretreatment levels of $\mathrm{HO}-1$ cannot be used to predict the outcome of TB therapy, but the extent of HO-1 expression measured at the end of therapy does reflect outcome. To quantitatively assess the accuracy of HO-1 levels at week 20 of therapy in discriminating successful treatment from failure or relapse, we performed receiver operator characteristics (ROC) curve analysis. We confirmed that HO-1 levels at the end of anti-TB treatment exhibited high accuracy in classifying treatment outcomes (area under the curve: 0.81 , sensitivity: $90 \%$, specificity $67.4 \%, p=0.003$; Figure S1 in Supplementary Material).

Additional analysis revealed that within the entire study population, HO-1 plasma concentrations did not differ between patients presenting with cavitary or non-cavitary disease (Figure 2D). Patients with non-cavitary disease exhibited a gradual decrease in HO-1 levels upon treatment initiation, while those with cavitary disease displayed significant changes in HO- 1 concentrations only at the end of therapy. Prior to therapy, circulating concentrations of HO-1 were not different between patients classified as having mild or extensive radiographic disease (Figure 2E). Moreover, treatment-induced reductions in HO-1 levels were independent of the radiographic extent (Figure 2E).

\section{HIV Disease Progression Affects HO-1 Expression in TB Patients}

In our patient cohort, $75(57.7 \%)$ patients were coinfected with HIV-1 (Table 1). Of those, 48 (64\%) were antiretroviral therapy (ART) naïve. Of note, $\mathrm{HO}-1$ concentrations in plasma were significantly higher in HIV-coinfected than in HIV-1uninfected patients before treatment initiation (Figure 2F). Although HO-1 levels substantially decreased upon treatment initiation irrespective of HIV status, HIV-coinfected patients had higher values than those of uninfected subjects at the end of TB therapy (Figure $2 \mathbf{F}$ ). These results suggest that HIV-1 coinfection may influence the regulation of HO-1 expression in TB patients. We next tested whether HIV-1-driven immunosuppression is associated with increased HO-1 expression in $\mathrm{TB}$ patients. In $\mathrm{TB} / \mathrm{HIV}$ coinfected patients, plasma levels of $\mathrm{HO}-1$ negatively correlated with $\mathrm{CD}^{+}{ }^{+} \mathrm{T}$-cell counts (Figure 3A) before anti-TB treatment. Individuals who were 
A

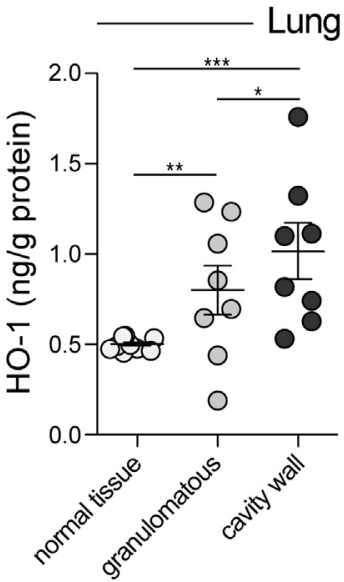

Lung homogenate

Plasma

Model 1: Iung cavitation
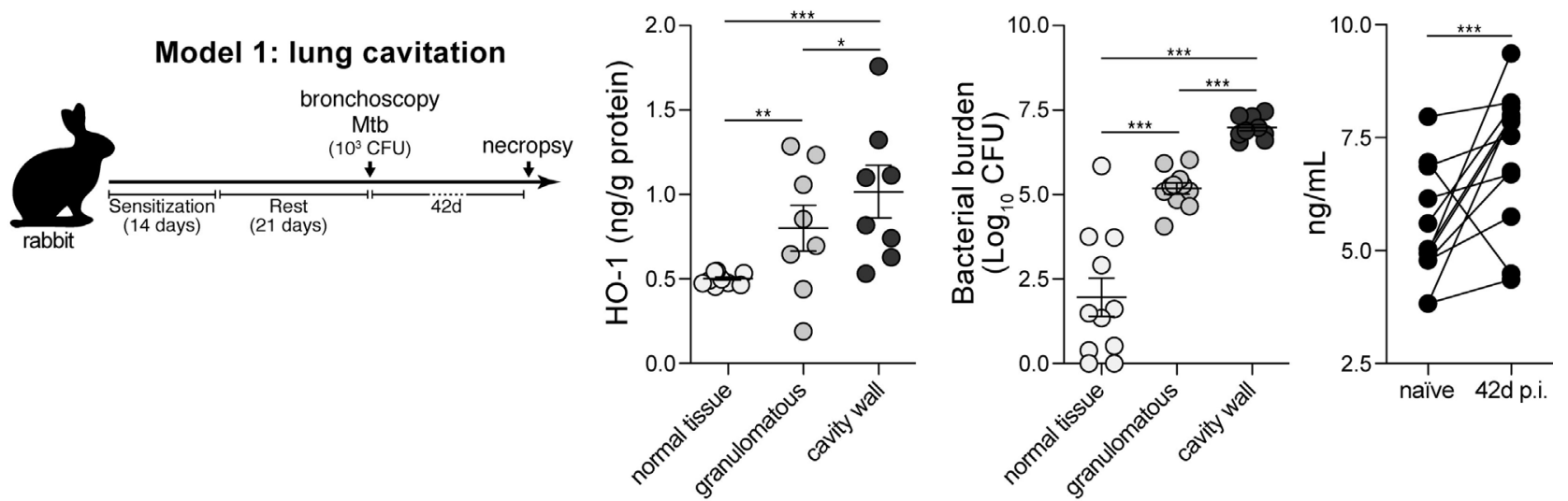

B

Model 2: full treatment

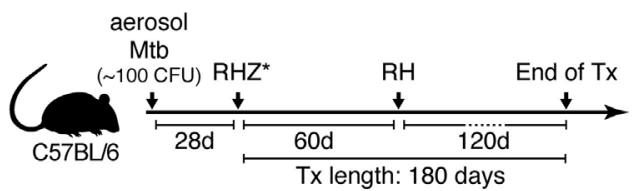

${ }^{*} \mathrm{R}$ : Rifampin H: Isoniazid Z: Pyrazinamide

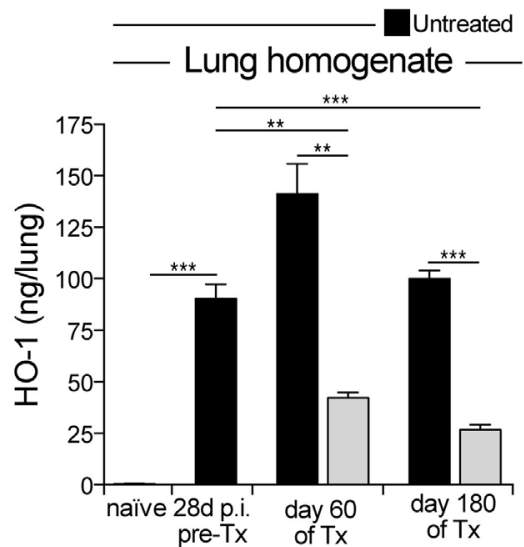

$\square \mathrm{RHZ} / \mathrm{RH}$ treated
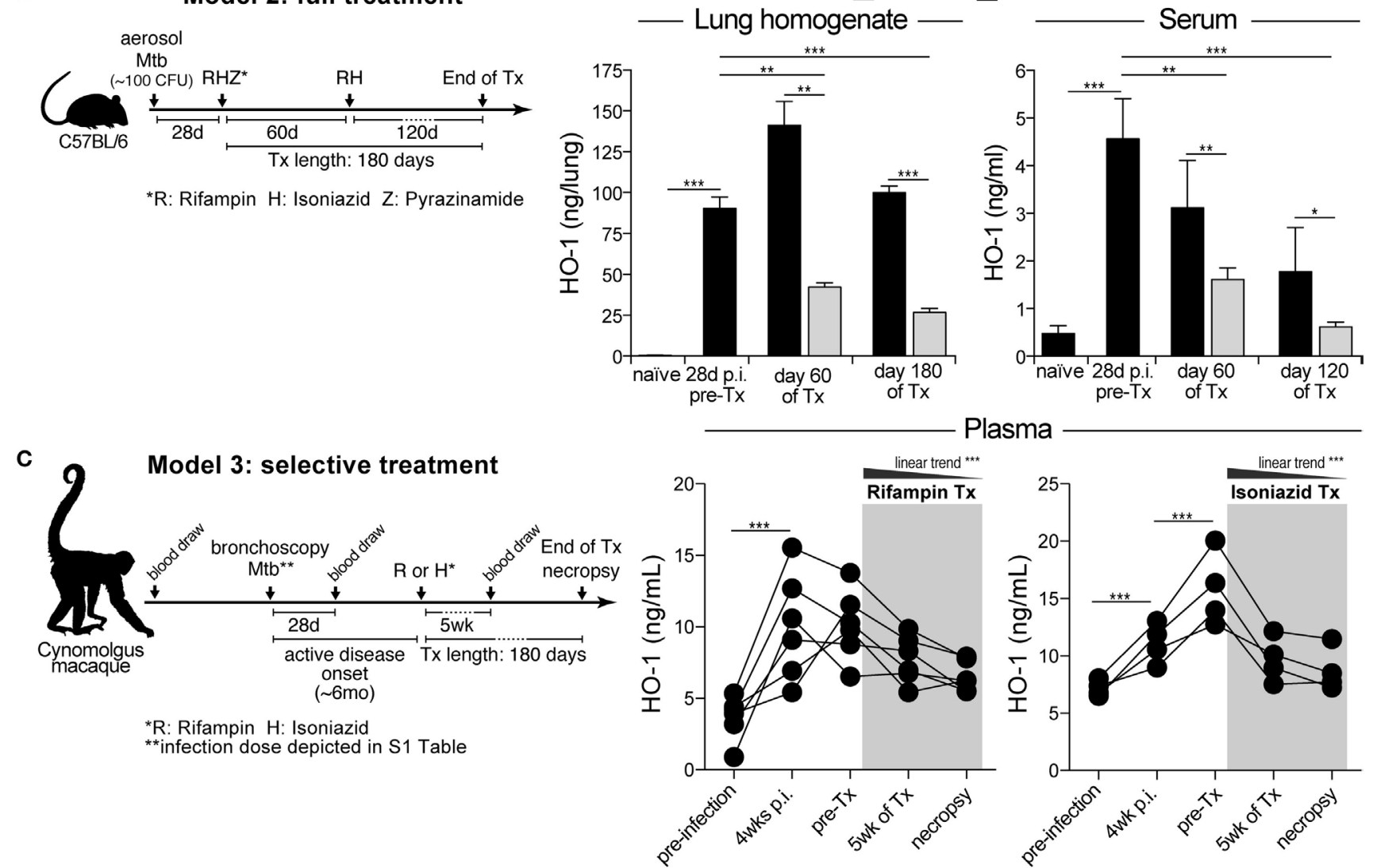

\section{Plasma}

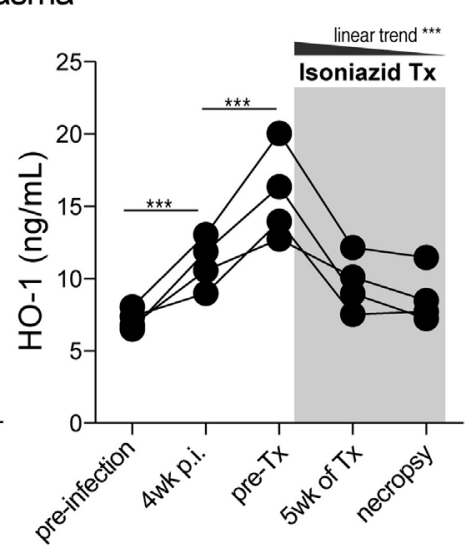

FIGURE 1 | Experimental Mycobacterium tuberculosis (Mtb) infection in vivo induces heme oxygenase-1 (HO-1) expression in lungs and blood, which is reversed during antitubercular therapy. (A) HO-1 protein expression was quantitatively assessed in lung homogenates and in plasma samples from rabbits bronchoscopically infected with Mtb by ELISA. (B) HO-1 levels in lung homogenates and serum samples were measured in a tuberculosis treatment model using mice infected with a low dose of Mtb. Graph shows representative data from different experiments using 4 mice in each time point per experimental group (total animal number $=8-12$ mice per group). (C) Prospective changes in $\mathrm{HO}-1$ concentrations in plasma were evaluated in non-human primates experimentally infected with $M$ tb and treated with rifampin or isoniazid. Data were analyzed using the Mann-Whitney $U$ test (two unmatched groups), the Kruskal-Wallis test with Dunn's multiple comparisons or linear trend ad hoc tests (more than two groups) or the Wilcoxon matched-pairs test $\left({ }^{\star} p<0.05,{ }^{* \star} p<0.01,{ }^{\star \star \star} p<0.0001\right)$.

highly immunosuppressed $\left(\mathrm{CD} 4^{+} \mathrm{T}\right.$-cell count $<100$ cells $\left./ \mu \mathrm{L}\right)$ exhibited increased HO-1 concentrations compared to those who were not (Figure 3B). Patients who were on ART at week 0 of ATT had lower HO-1 levels than those who were ART-naïve
(Figure 3C). In addition, HO-1 levels positively correlated with HIV-1 viral load (Figure 3D). These findings suggest that $\mathrm{HIV}-1$ coinfection causes further increases in HO-1 expression in TB patients. 

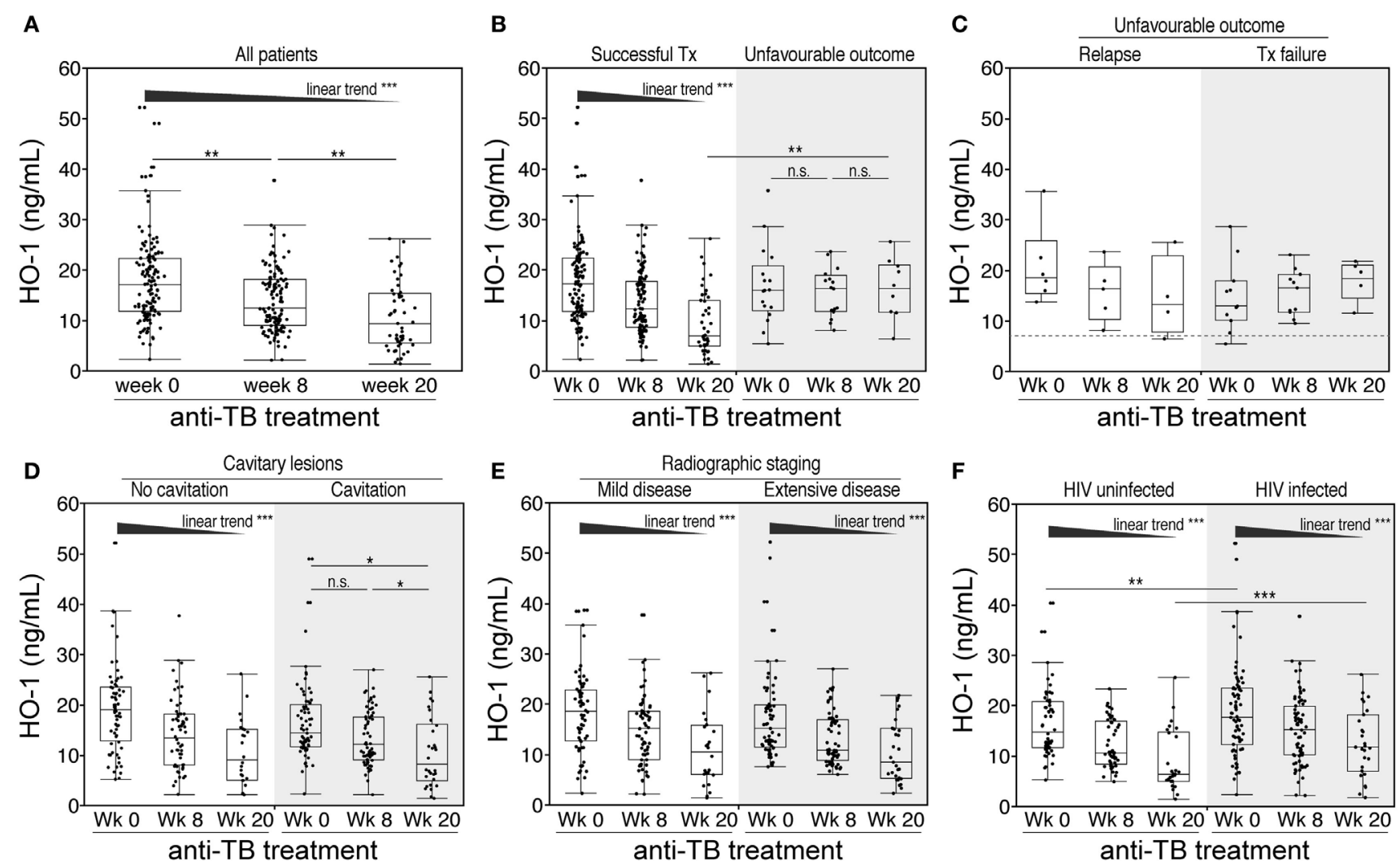

FIGURE 2 | Changes in plasma concentrations of heme oxygenase-1 (HO-1) in pulmonary tuberculosis (TB) patients undergoing antitubercular treatment. (A) Plasma levels of $\mathrm{HO}-1$ were prospectively assessed in samples from 130 patients with pulmonary TB before and at the indicated time points after anti-TB treatment initiation. (B,C) $\mathrm{HO}-1$ levels in patients with successful outcome and in those who had unfavorable outcomes (treatment failure or relapse). $\mathrm{HO}-1$ levels at different time points upon anti-TB treatment were also stratified by the presence of cavitary lesions (D), extensive disease on X-ray at pretreatment (E), or HIV infection status (F). Data were analyzed using Friedman matched pairs with Dunn's multiple comparisons test as well as non-parametric linear trend post hoc test.

TABLE 1 | Characteristics of study participants.

\begin{tabular}{|c|c|c|c|c|}
\hline Characteristic & All & HIV-1 infected & HIV-1 uninfected & $p$-Value \\
\hline$N$ & 130 & 75 & 55 & \\
\hline Male-no. (\%) & $73(56.1)$ & $32(42.7)$ & $41(74.5)$ & 0.0003 \\
\hline Median age-years (IQR) & $35.1(30-44)$ & $35.1(10.5-42.1)$ & $35.4(27.5-51)$ & 0.994 \\
\hline Median BMI-kg/m² (IQR) & 21 (19-23) & $22(20-24)$ & $20(19-23)$ & 0.035 \\
\hline \multicolumn{5}{|l|}{ Comorbidities - no. (\%) } \\
\hline Diabetes mellitus & $9(6.9)$ & $4(5.3)$ & $5(9.1)$ & 0.489 \\
\hline Smoking history & $60(46.1)$ & 29 (38.6) & $31(56.4)$ & 0.048 \\
\hline AFB smear -no. (\%) & & & & 0.015 \\
\hline Negative/scanty & $46(35.4)$ & $34(45.4)$ & $12(21.8)$ & \\
\hline $1+$ & $24(18.5)$ & $15(20)$ & $9(16.4)$ & \\
\hline $2+$ & $28(21.5)$ & $13(17.3)$ & $15(27.3)$ & \\
\hline $3+$ & $32(24.6)$ & $13(17.3)$ & $19(34.5)$ & \\
\hline Extensive radiological disease-no. (\%) & $91(70.0)$ & $41(54.6)$ & $50(90.9)$ & $<0.0001$ \\
\hline Cavitation - no. (\%) & $67(51.5)$ & $33(44)$ & $34(61.8)$ & 0.049 \\
\hline Culture conversion at week $8-$ no. (\%) & $75(57.7)$ & $49(65.3)$ & $26(47.3)$ & 0.070 \\
\hline Death-no. (\%) & $5(3.8)$ & $5(6.7)$ & 0 & 0.058 \\
\hline
\end{tabular}

Frequency data between HIV-infected and -uninfected active TB patients were analyzed using the Fisher's exact test or the Pearson's chi-square test. Continuous variables were compared using the Mann-Whitney $U$ test. $p$-Values were adjusted for multiple comparisons. 
TB/HIV-1 co-infected patients (week 0 of anti-TB treatment)

A

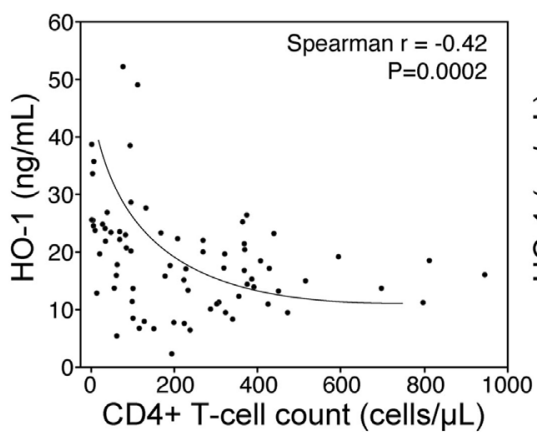

B

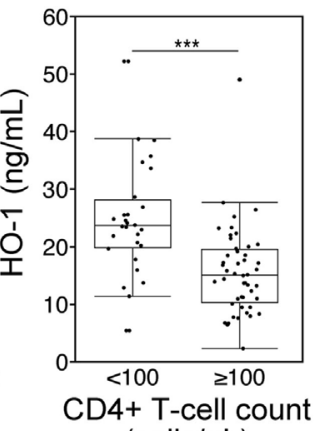

C

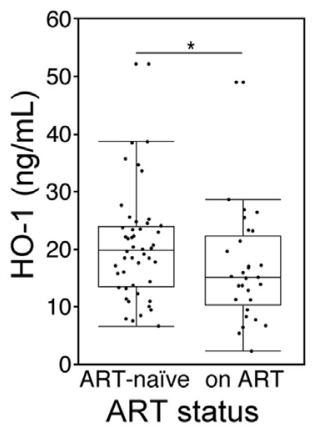

D detection limit

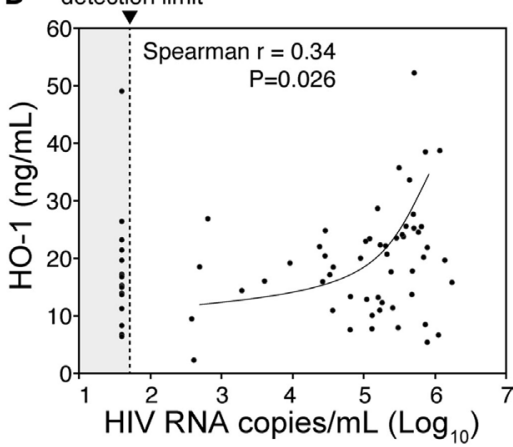

FIGURE 3 | HIV-1 coinfection and degree of immunosuppression relates to heme oxygenase-1 (HO-1) concentrations in plasma from tuberculosis (TB) patients prior to antitubercular treatment. (A) Spearman correlation analysis of HO-1 levels in plasma by CD4+ ${ }^{+}$-cell counts in $\mathrm{HIV}^{-i n f e c t e d ~ p a t i e n t s ~ b e f o r e ~}$ initiation of antibubercular therapy. (B) HIV-infected patients were further classified based on CD4+ $\mathrm{T}$-cell counts and $\mathrm{HO}-1$ levels. (C) HO-1 levels were also compared between antiretroviral therapy (ART)-naiive patients and those who were on ART before TB treatment. In panels (B,C), data were compared using the Mann-Whitney $U$ test. (D) Spearman correlation analysis of $\mathrm{HO}-1$ levels in plasma with HIV viral load at pretreatment in HIV-infected patients.

\section{Induction of HO-1 in Human and Mouse Macrophages Infected with Mtb Requires NADPH-Derived ROS Production}

The results presented above demonstrate that HO-1 is induced upon $M t b$ infection in different experimental models in vivo as well as in patients with active disease. To further delineate the molecular mechanisms to induce HO-1 in the context of $M t b$ infection, we employed an in vitro system using mouse and human macrophages infected with $M t b \mathrm{H} 37 \mathrm{Rv}$ strain (10). We chose macrophages because this cell type is known to be an important source of HO-1 in several disease models including human and murine mycobacterial infections (29). In other HO-1 induction systems, expression of the enzyme is thought to require translocation to the nucleus of the transcription factor known as nuclear factor erythroid-derived 2-like 2 (NRF-2) (14). We found that this is also true for HO- 1 induction by $M t b$ infection as bone marrow-derived macrophages (BMDMs) from mice genetically lacking NRF-2 were unable to produce HO-1 following bacterial exposure (Figures $\mathbf{4 A}, \mathbf{B}$ ). In our in vitro system, the results obtained with $\mathrm{HO}-1$ protein quantification reflected the degree of HMOX1 messenger RNA (mRNA) expression (Figure S2 in Supplementary Material).

Several signaling pathways have been described to be associated with HO-1 expression, including classical patternrecognition pathways (38), interleukin-10 (IL-10) (39), and type I interferon (40). Remarkably, induction of both nuclear NRF-2 translocation and $\mathrm{HO}-1$ protein expression was not affected in $M t b$-infected BMDM from mice lacking MyD88, TRIF, IL-10, interferon alpha and beta receptor subunit 1 (IFNAR), or ferritin heavy chain (FTH1) (Figures 4C-L). These observations reveal that HO-1 induction driven by Mtb infection of macrophages involves a mechanism that is independent of numerous signaling pathways involved in molecular recognition of the pathogen and innate immune responses classically linked to TB pathogenesis.
We have recently described that patients with active pulmonary TB exhibit elevated circulating levels of oxidation products and lipid peroxidation compared with individuals with LTBI or uninfected controls, as well as in human macrophages infected with $M t b$ in vitro, demonstrating an important role for oxidative stress in this disease (11). In this study, we asked whether the oxidative stress triggered by $M t b$ infection might play a role in driving HO-1 expression. Indeed, treatment of $M t b$-infected human monocyte-derived macrophage cultures with Tempol, a potent antioxidant, caused a substantial reduction in HO-1 protein expression (Figure 5A, left panel).

In unstimulated conditions, NRF-2 has been described to interact with the protein Kelch-like ECH-associated protein 1 (Keap-1) in the cytosol of macrophages and other cell types. This interaction induces proteasome-mediated degradation of the former molecule. Upon stimulation, ROS is thought to interfere with the NRF-2/Keap-1 interaction, releasing the transcription factor, which then translocates to the nucleus to induce $\mathrm{HO}-1$ $(41,42)$. To test if the ROS requirement for HO-1 induction in our in vitro system involves a similar pathway, we incubated cultures of infected human monocyte-derived macrophages treated with Tempol in the presence of TAT-14, a peptide comprising a TATconjugated NRF-2 sequence known to interfere with the NRF-2/ Keap-1 interaction (43). Notably, HO-1 induction was restored when TAT-14 was added to the cultures of infected cells treated with Tempol (Figure 5A, right panel), without altering ROS production (Figure 5B). The restoration of HO-1 induction upon $M t b$ infection was reflected by increased activation of NRF-2 in nuclear extracts (Figure 5C). These findings demonstrate that ROS-mediated interference of NRF-2/Keap-1 interaction is a major pathway involved in the induction of HO- 1 by $M t b$ in murine macrophages.

The NADPH oxidase system is a major source of ROS in both physiological and pathological conditions (44). To test if NADPHderived ROS plays a role in Mtb-driven HO- 1 induction, we 

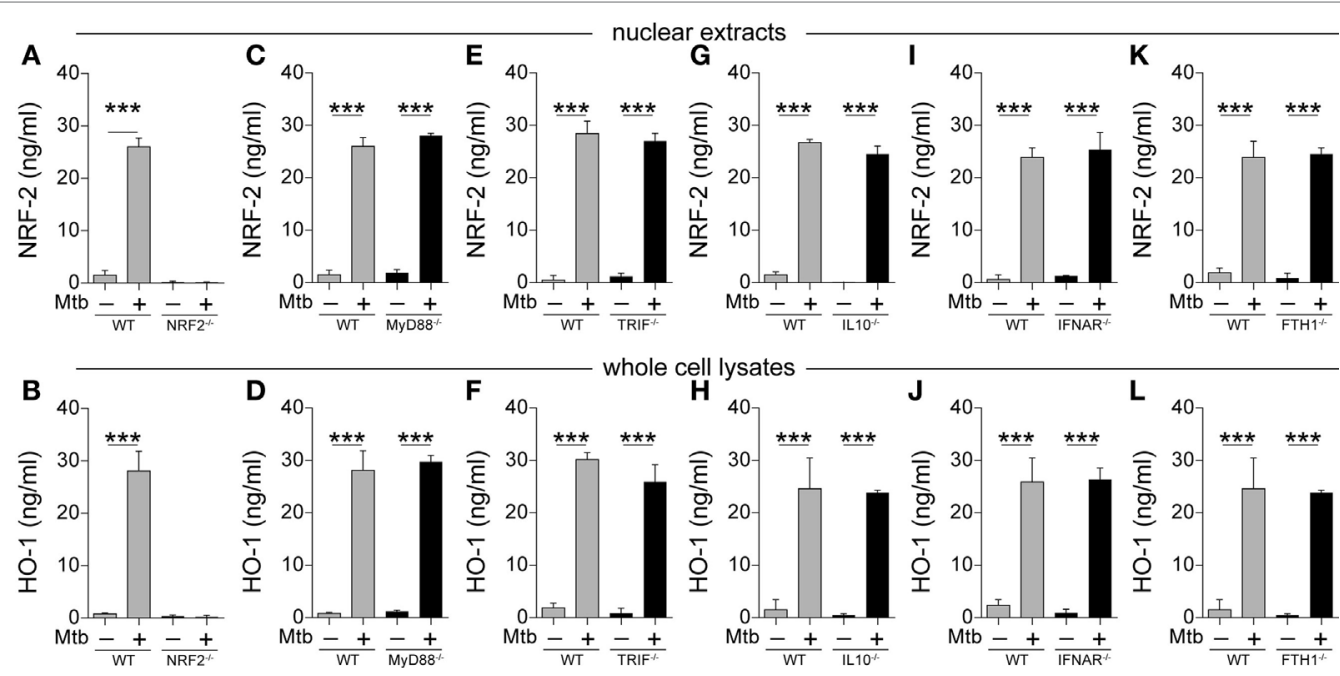

FIGURE 4 | Mycobacterium tuberculosis (Mtb) induction of heme oxygenase-1 (HO-1) protein expression in mouse macrophages is independent of multiple signaling pathways linked to innate immunity in tuberculosis. (A-L) Bone marrow-derived macrophages from mice genetically lacking expression of the indicated genes or their respective wild-type (WT) controls were infected with H37Rv Mtb (multiplicity of infection: 3 ) for 24 h. Protein expression of nuclear factor erythroid-derived 2-like 2 (NRF-2) and HO-1 was quantified in nuclear extracts and whole cells lysates, respectively, by ELISA. Bars and lines represent mean and SEM, respectively. Data are from at least three independent experiments using triplicate biological samples. Data were compared using the Wilcoxon matched-pairs test ( ${ }^{*} p<0.05,{ }^{* *} p<0.01,{ }^{\star * *} p<0.0001$ ). MyD88, myeloid differentiation primary response gene 88 ; TRIF, TIR domain-containing adapter-inducing interferon- $\beta$; IL-10, interleukin-10; IFNAR, interferon alpha and beta receptor subunit 1; FTH1, ferritin heavy chain 1.
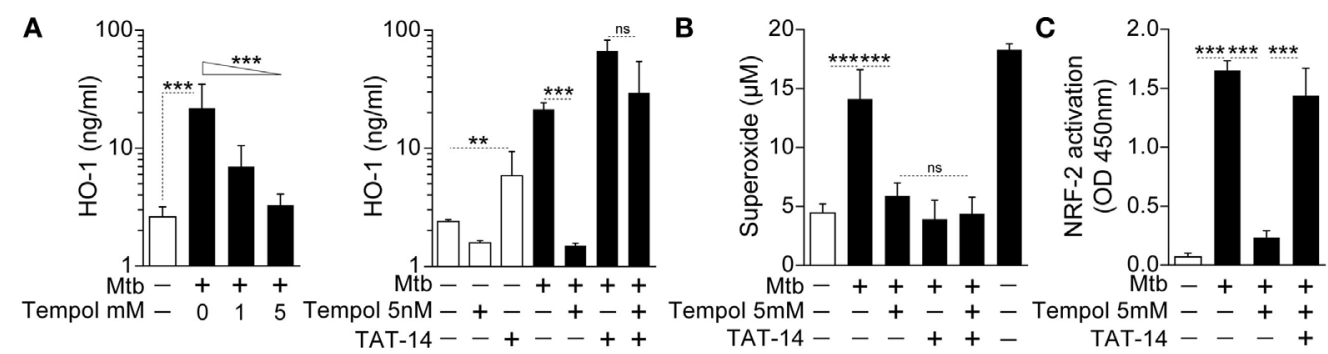

D

E

TAT-14 --++-
$\mathrm{H}_{2} \mathrm{O}_{2}--+$

TAT-14
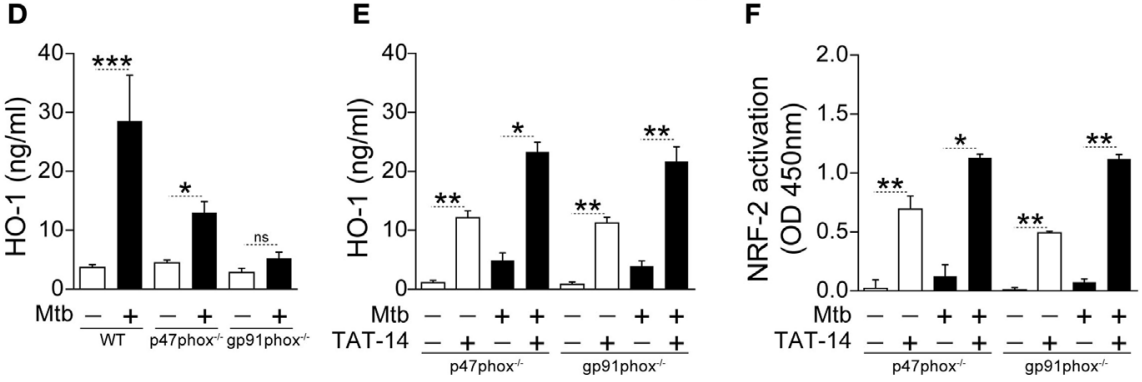

FIGURE 5 | NADPH-derived reactive oxygen species is required for induction of heme oxygenase-1 (HO-1) in macrophages infected with Mycobacterium tuberculosis (Mtb). (A) Human monocyte-derived macrophages were infected with Mtb H37Rv [multiplicity of infection (MOI): 3] in the presence or absence of an antioxidant (Tempol) for $24 \mathrm{~h}$ and $\mathrm{HO}-1$ levels were measured in whole cell extracts by ELISA. Right panel shows HO-1 levels in infected macrophages treated with Tempol in the presence of a TAT-conjugated NRF-2 sequence peptide that interacts with the Keap-1/NRF-2 complex (TAT-14, 50 MM). (B) Generation of superoxide anions was quantified in cell supernatants of cultures shown in panel (A). $\mathrm{H}_{2} \mathrm{O}_{2}$ was used as positive control. (C) Activation of the $\mathrm{HO}-1$ transcription factor NRF-2 in nuclear extracts was quantitatively assessed $12 \mathrm{~h}$ after infection using a colorimetric DNA-binding ELISA kit. (D) Bone marrowdifferentiated macrophages were prepared from wild type (WT), p47phox ${ }^{-/-}$, or gp91 phox ${ }^{-/}$(deficient in two distinct subunits of the NADPH oxidase system) mice and infected in vitro with $\mathrm{Mtb}(\mathrm{MOI}: 3)$ for $24 \mathrm{~h}$. $\mathrm{HO}-1$ levels were then assessed in whole cell extracts by ELISA. (E,F) HO-1 levels in cell extracts as well as activation of NRF-2 in nuclear extracts were quantified in macrophage cultures in the presence of TAT-14 (50 $\mu \mathrm{M})$. Data are from at least three experiments using cells from a total of up to six healthy donors. (D-F) Three independent experiments were performed, with samples run in triplicates. Data from different biological groups were analyzed using the Kruskal-Wallis test, with the Dunn's multiple-comparison test, whereas matched analyses were performed using the Wilcoxon matched-pairs test $\left({ }^{\star} p<0.05,{ }^{\star \star} p<0.01,{ }^{\star \star \star} p<0.001\right)$. ns, non-significant; NRF-2, nuclear factor erythroid-derived 2 -like 2. 
infected BMDM from mice deficient in either one of the two main NADPH-oxidase subunits (p47Phox and gp91Phox), which lack fully functional NADPH-oxidase activity $(45,46)$. Importantly, $M t b$-induced HO-1 expression was markedly diminished in the genetically deficient mice compared with the wild-type (WT) animals (Figure 5D). In these experiments, the $\mathrm{p} 47 \mathrm{Phox}^{-1-}$ mice and their respective WT controls were from C57BL/6NTac genetic background, whereas the gp91 $\mathrm{Phox}^{-/-}$animals were from the C57BL/6J background. The latter genetic background has been associated with a spontaneous mutation that results in mitochondrial redox abnormalities (47), which could have influenced the HO-1 production in vitro. However, the pattern of $\mathrm{HO}-1$ production in the experimental conditions was very similar to that observed in mice from the C57BL/6NTac genetic background. In addition, as observed with Tempol-treated human macrophages (Figure 5A), TAT-14 addition restored both HO-1 expression and NRF-2 activation (Figures 5E,F, respectively) to the NADPH oxidase-deficient macrophage cultures from both genetic backgrounds. These results support the hypothesis that $M t b$ drives HO-1 expression in infected macrophages by inducing NADPH-derived ROS, which inhibits NRF2/Keap1 interaction.

\section{Mtb-Associated Oxidative Stress and HO-1 Production Are Dependent on ESAT- 6 Expression}

Many pathological effects observed in cells infected with $M t b$ are related to bacterial production of the virulence factor ESAT-6, a protein that is secreted into the host cell cytosol (48). We have previously reported that $M t b$ lacking ESAT- 6 failed to induce robust $\mathrm{HO}-1$ expression in both human and murine macrophages (10). Experiments were next performed to determine whether the role of ESAT-6 in HO-1 induction involves the oxidative stressdependent pathway described above. We found that infection of human macrophages with an H37Rv mutant lacking ESAT-6 was associated with a decreased extracellular and intracellular ROS response (Figure 6A). This reduction in ROS production was linked to reduced concentrations of malondialdehyde (MDA), a marker of lipid peroxidation, and of 8-hydroxy2 -deoxyguanosine (8-OH-dG), a readout of oxidative DNA damage (Figure 6B). We next delivered recombinant ESAT-6 protein fused with the $\mathrm{N}$-terminal fragment of the lethal factor of Bacillus anthracis $(10,49)$ into the cytosol of macrophages infected with ESAT-6-deficient $M t b$. Notably, delivery of ESAT-6 into macrophages restored ROS production (Figure 6A) and was further associated with augmented lipid peroxidation and oxidative DNA damage (Figure 6B), at levels comparable to that induced by the control WT H37Rv strain. As expected, the decreased HO-1 expression observed in macrophages infected with ESAT-6-deficient $M t b$ was associated with reduced NRF-2 activation in nuclear extracts (Figure 6C), and these defects were corrected when macrophage cultures were treated with TAT-14 (Figure 6C). Together, these findings support a link between ESAT-6 expression and ROS-induced activation of NRF-2 in the triggering of HO- 1 production by $M t b$ in human macrophages.

Previous studies in both murine BMDMs and RAW264.7 cells found that exposure to soluble recombinant ESAT-6 protein
A

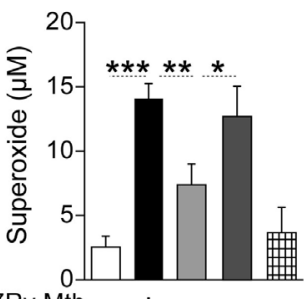

H37Rv Mtb -+-$\triangle$ ESAT6 Mtb $- \pm++\overline{+}-$ PA-Lfn-ESAT6-His6 - - ++

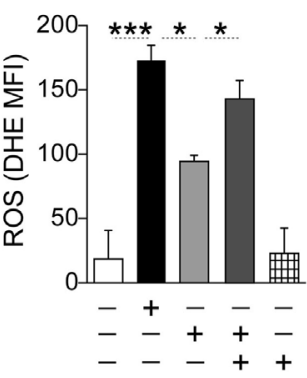

B
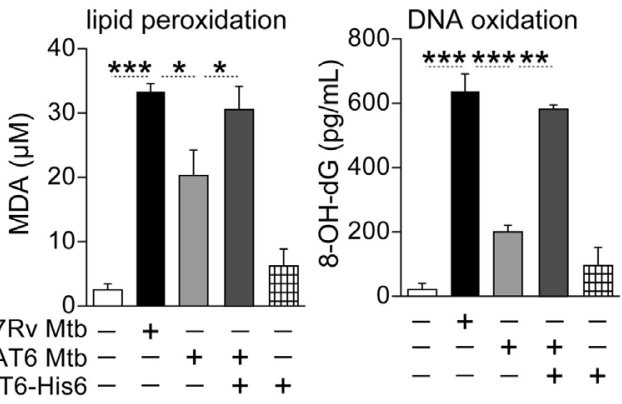

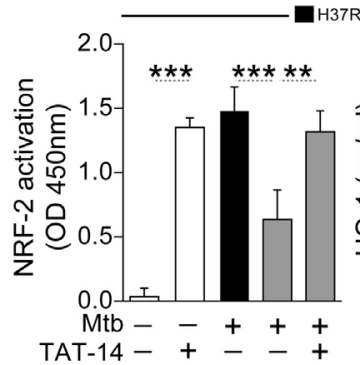

FIGURE 6 | ESAT6-mediated heme oxygenase-1 (HO-1) induction in Mycobacterium tuberculosis (Mtb)-infected macrophages is associated with reactive oxygen species (ROS)-dependent release of NRF-2 to the nucleus. (A) Human monocyte-differentiated macrophages were infected with Mtb H37Rv or an H37Rv mutant strain lacking ESAT-6 expression (both at multiplicity of infection: 3 ) for $24 \mathrm{~h}$. Cells infected with the ESAT-6-deficient Mtb strain were treated with $20 \mu \mathrm{g} / \mathrm{mL}$ of the fusion protein Lfn-ESAT- 6 with the anthrax-protective antigen cytosolic delivery system.

Generation of superoxide anions was quantified in cell supernatants using a colorimetric assay, while intracellular ROS production was assessed by flow cytometry. Results are plotted as histograms where the mean fluorescence intensity (MFI) was compared between the experimental groups. (B) Lipid peroxidation and oxidative DNA damage were assessed as described in Section "Materials and Methods." (C) Activation of NRF-2 in nuclear extracts (12 h postinfection) and of $\mathrm{HO}-1$ levels in whole cell lysates (24 h postinfection) were determined in cultures of infected macrophages in the presence or absence of a TAT-conjugated NRF-2 sequence peptide that interacts with the Keap-1/NRF-2 complex (TAT-14, $50 \mu \mathrm{M})$. Data are from at least three independent experiments using cells from a total of up to six healthy donors. Data were compared using the Wilcoxon matched-pairs test $\left({ }^{\star} p<0.05,{ }^{\star \star} p<0.01,{ }^{\star \star *} p<0.0001\right)$. MDA, malondialdehyde; NRF-2, nuclear factor erythroid-derived 2-like 2; 8-OH-dG, 8-hydroxy-2'deoxyguanosine.

triggers HO-1 expression via induction of the microRNA 155 (miR-155) (50). We therefore tested the participation of miR-155 in our in vitro system by assessing NRF-2 nuclear translocation and $\mathrm{HO}-1$ production in miR-155KO murine macrophages 
(Figure S3 in Supplementary Material). Our experiments revealed that $M t b$-infected BMDMs from miR-155KO mice failed to induce HO-1 production while exhibiting NRF-2 quantification in nuclear extracts similar to that observed in WT cells. Such diminished HO-1 production detected in cultures from miR$155 \mathrm{KO}$ cells was not reverted by treatment with TAT-14 (Figure S3 in Supplementary Material). These findings suggest that miR-155 is critical to induce $\mathrm{HO}-1$ production in $M t b$-infected macrophages by a distinct pathway that does not involve interference with NRF-2/Keap-1 interaction.

\section{DISCUSSION}

Heme oxygenase- 1 production is induced during the course of several infectious diseases as well as inflammatory disorders and may play beneficial or detrimental role for the host depending on the stimulus and context $(29,30,40,51-57)$. Mtb infection, in particular, induces a robust production of the enzyme detected in plasma $(27,31,32)$ suggesting its potential use as a biomarker for TB. Indeed, our previous studies on a cohort of South Indian HIV-negative TB patients revealed a correlation between $\mathrm{HO}-1$ levels and active disease and demonstrated a reduction in the enzyme following successful antibiotic treatment (27). In this study, we have confirmed the association of circulating levels of HO-1 with $M t b$ infection in three different animal models and in the case of the rabbit correlated enzyme expression with pulmonary pathology. Moreover, we were able to replicate in mice and $\mathrm{NHP}$ the reduction in $\mathrm{HO}-1$ levels observed in patients following chemotherapy.

To further examine the utility of $\mathrm{HO}-1$ as an accurate biomarker of active $M t b$ infection in humans and to assess the impact of HIV coinfection on enzyme expression, we performed a prospective analysis of HO-1 levels in a South African cohort of TB and HIV-TB patients prior to and at an early time point ( 8 weeks) as well as at 20 weeks post-ATT. In agreement with our previous findings, a major (approximately threefold) decrease in HO-1 levels was observed in all successfully treated patients after 20 weeks of chemotherapy. More importantly, decreased enzyme levels were also evident at 8 weeks posttreatment, but only in those patients lacking cavitary lesions who went on to clear their infections. These findings suggest that measurement of HO-1 levels could be a useful correlate of successful therapy at the conclusion of conventional treatment, but is unlikely to be a reliable early predictor of treatment outcome. Whether those individuals with lowered HO-1 levels at early time points would still clear their infections if therapy was withdrawn is unclear and was not tested in this study.

Approximately $58 \%$ of the TB patients in our cohort were HIV-1 coinfected allowing us to evaluate the influence of HIV coinfection on HO-1 expression. Although it is unclear from previous studies whether HIV infection results in elevated plasma HO-1 levels, the virus has been reported to trigger downmodulation of HO-1 expression in brain tissue as well as in infected monocytederived macrophages $(58,59)$. Our results show that HIV/Mtb coinfected patients display higher $\mathrm{HO}-1$ levels, which inversely correlated with $\mathrm{CD}^{+}$T-cell counts and positively correlated with viral load, indicating that HIV coinfection quantitatively enhances HO-1 expression in TB patients. Alternatively, the increased $\mathrm{HO}-1$ found in $\mathrm{HIV} / \mathrm{TB}$ patients may merely reflect the increase in enzyme levels resulting from the expected defective control of $M t b$ in these individuals $(60,61)$, although such an effect was not evident in the sputum smears performed in the cohort studied here (Table 1). Moreover, as described above for $M t b$ monoinfected patients, successful anti-TB treatment also resulted in reduced plasma HO-1 levels in HIV coinfected individuals. Although these observations suggest that $M t b$ is the force driving HO-1 expression in HIV coinfected patients, further studies involving individuals with HIV monoinfection and comparing the effects of antiretroviral treatment versus anti-TB therapy in coinfected subjects are needed to test this hypothesis. Another hypothesis to explain heightened HO-1 levels in HIV/ $\mathrm{TB}$ coinfected patients is that HIV induces pyroptosis in $\mathrm{CD}^{+}$ T-cells (62), subsequently leading to massive inflammation and release of cytosolic content. This would potentially explain why $\mathrm{HIV} / \mathrm{TB}$ coinfected patients still display increased $\mathrm{HO}-1$ levels at the end of anti-TB treatment as well as the observed reductions in serum HO-1 in patients on ART compared to ART-naïve individuals.

The mechanisms through which $M t b$ infection induces HO-1 expression in vivo or for that matter in infected cells are not completely understood. In the case of cultured macrophages, we and others have demonstrated a role for the $M t b$ virulence factor ESAT-6 in HO-1 induction. While our previous experiments with primary human macrophages indicated a requirement for live infection with ESAT-6 expressing bacteria and cytosolic delivery of the protein (10), Kumar et al. studying both murine BMDMs and RAW264.7 cells found that exposure to soluble recombinant ESAT-6 protein alone was sufficient to trigger $\mathrm{HO}-1$ expression (50). Analyzing the mechanism responsible for $\mathrm{HO}-1$ induction, the latter authors identified a major role for the micro RNA miR-155 and Bach-1 in the transcriptional regulation of HO-1. In other studies, soluble ESAT-6 has been shown to stimulate miR-155 through a TLR2, MyD88-dependent pathway (63).

The data shown in this report demonstrate a role for ROS in the ESAT-6-dependent induction of HO- 1 by $M t b$. This pathway appears to be independent of TLR, MyD88, as well as TRIF signaling and thus apparently distinct from the miR-155 pathway described by Kumar et al. (50). A link between oxidative stress and HO-1 production has been documented previously in other systems (64-66), and we and others have shown that $M t b$ infection is a potent stimulus of ROS production $(11,67)$. Importantly, we show in this study that ESAT-6-dependent stimulation of ROS promotes HO-1 production by inducing the release and nuclear translocation of the transcription factor NRF-2, which normally exists as a cytosolic complex with Keap-1 $(41,42)$. We speculate that the activation of NRF-2 by this pathway is responsible for the transcription of HO-1 induced by $M t b$ in macrophages. Keap-1 has previously been identified as a sensor of oxidative stress (68) and been linked to HO-1 expression through the NRF-2 pathway studied here $(41,42,69)$. Our findings are the first to formally establish the participation of this mechanism in the induction of $\mathrm{HO}-1$ by $M t b$. In future experiments, it will be important to identify the pathway by which intracellular ESAT-6 is recognized and triggers ROS production in infected cells. In addition, further 
studies are needed to define the relationship of the ESAT-6/ ROS/NRF-2/HO-1 pathway described here with the previously reported ESAT-6-driven miR-155-dependent mechanism that de-represses Bach-1-mediated suppression of HO-1 transcription. Conceivably, both mechanisms could coregulate expression of the enzyme in different contexts. Indeed, our experiments demonstrating that $M t b$-infected BMDM from miR-155 KO mice fail to induce HO-1 production by a mechanism dependent on NRF-2 nuclear translocation reinforce the hypothesis that there are likely other pathways by which $M t b$ triggers HO-1 expression in macrophages.

While the data presented here add new information on the expression of HO-1 in TB infection and disease as well as the mechanisms underlying the induction of this important biomarker, the precise physiological role played by the enzyme in the immunopathogenesis of $M t b$ infection remains unclear. The biological mechanisms underlying HO-1 expression in plasma of patients is not clearly understood. HO-1 is described as a cytosolic enzyme expressed in many cell types and tissues. Our results from plasma measurements of this enzyme led us to hypothesize that in pulmonary TB patients, the increased $\mathrm{HO}-1$ observed in plasma derives from injured tissues, a concept that would explain the strong association of $\mathrm{HO}-1$ with bacterial burden (27). Furthermore, as discussed earlier, previous results from our group and others have shown that pharmacological inhibition of HO- 1 activity can reduce $M t b$ loads in vitro in infected human macrophages (29) as well as in vivo in an experimental murine model of TB and result in accelerated bacterial clearance when used in conjunction with antibiotics (30). Nevertheless, there is contrary data to suggest that the primary role of $\mathrm{HO}-1$ induction is host protection (23) consistent with the known antioxidant and cytoprotective functions of the enzyme. Further research is clearly necessary to resolve this controversy. Regardless, continued dissection of the mechanisms underlying HO-1 induction in $M t b$ infection will be important in revealing potential targets for either suppressing or enhancing this response as an approach to therapeutic intervention and for further understanding the precise parameters that $\mathrm{HO}-1$ reflects when used as a biomarker for $\mathrm{TB}$ disease and treatment.

\section{MATERIALS AND METHODS}

\section{Patient Population and Clinical Procedures}

Patients with GeneXpert Mtb/RIF-confirmed rifampinsusceptible pulmonary TB were recruited at the Ubuntu HIV/ TB Clinic, Site B, Khayelitsha, South Africa, as part of a prospective cohort study. Detailed sociodemographic data, past TB treatment history, and comorbidity data were collected. At the study baseline, HIV testing (Abbott Architect HIV Ag/Ab Combo test), $\mathrm{CD}^{+}{ }^{+} \mathrm{T}$ lymphocyte count, and HIV-1 viral load quantification were performed. Extensive disease on chest X-ray was performed by experts and noted as either involvement of $>1$ lung or involvement of $\geq 1$ out of 3 (upper, mid, or lower) zones per lung. Antitubercular drugs were provided in a 4-drug fixed-dose combination supplied by the National Tuberculosis
Control Programme [Rifafour e-275 (Sanofi-Aventis) or Ritib (Aspen, South Africa)]. Each tablet contained rifampin at $150 \mathrm{mg}$, isoniazid at $75 \mathrm{mg}$, pyrazinamide at $400 \mathrm{mg}$, and ethambutol at $275 \mathrm{mg}$. Weight band-based dosing was used in line with WHO guidelines (70). Patients weighing 38-55, >55-70, and $>70 \mathrm{~kg}$ were given doses of 3, 4, and 5 tablets, respectively. Anti-TB drugs were administered 7 days/week, along with $25 \mathrm{mg}$ pyridoxine. Clinical care remained the responsibility of the Site B TB clinic. Unfavorable treatment outcomes were defined as failure or relapse. Failure was any TB patient who was culture positive at 5 months or more after starting treatment. Relapse was defined as a TB patient who was declared cured or treatment completed by a physician, but who reports back to the health service and is now found to be sputum culture positive. EDTA plasma samples were collected before initiation of ATT as well as at month 2 and 5 of treatment. Samples were frozen at $-80^{\circ} \mathrm{C}$ until use in the immunoassays.

\section{Rabbit Lung Cavitation Model}

The lung and plasma samples employed were from infected animals described in previously published papers $(33,34)$. In these studies, female New Zealand white rabbits, weighing 3-3.5 kg (Covance Research Products, Gaithersburg, MD, USA), were sensitized with five $0.2 \mathrm{ml}$ subcutaneous injections of $1 \times 10^{7}$ heat-killed Mycobacterium bovis, emulsified 1:1 in Freund's adjuvant. Twenty-one days after sensitization, skin test reactivity was determined by injection of 5 IU of purified protein derivative (Tubersol; Sanofi-Aventis, Bridgewater, NJ, USA) and measured at $48 \mathrm{~h}$. For infection, rabbits were anesthetized by intramuscular administration of ketamine $(10 \mathrm{mg} / \mathrm{kg})$ and xylazine $(20 \mathrm{mg} / \mathrm{kg})$ prior to endotracheal tube intubation. A 3.0-mm flexible Pentax FB-8V pediatric bronchoscope (Pentax, Montvale, NJ, USA) was guided into 1 lower lobe, and $400 \mu \mathrm{L}$ of $7.5 \times 10^{3} \log$-phase $M t b$ strain H37Rv was inoculated via catheter. At day 42 postinfection, EDTA plasma specimens were collected from the central ear artery. Rabbits were euthanized following anesthesia by intravenous injection of Euthasol (Virbac, Fort Worth, TX, USA). Different lung regions presenting with cavitation (cavity wall), granulomatous lesions, or normal lung tissue were dissected for protein analysis and snap frozen in liquid nitrogen as previously described $(33,34)$. In addition, $3 \mathrm{~mm}$ punch biopsies (six/tissue/ animal) from each dissected lung region were weighed and then homogenized in phosphate-buffered saline (PBS) prior to $\mathrm{Mtb}$ CFU enumeration on $7 \mathrm{H} 11$ selective medium (33). The remaining lung lobe ( $>90 \%$ by mass) was weighed and then homogenized, using a Polytron homogenizer (Kinematica, CH), in $40 \mathrm{~mL}$ PBS prior to CFU enumeration by serial dilution on selective $7 \mathrm{H} 11$ medium.

\section{Murine Model of Pulmonary TB}

C57BL/6J mice between 6 and 8 weeks of age were obtained from National Institute of Allergy and Infectious Diseases (NIAID) through a supply contract with Taconic Farms (Germantown, NY, USA). All mice were bred and housed at Association for the Assessment and Accreditation of Laboratory Animal Care accredited Biosafety level 2 and 3 facilities at the NIAID, NIH, following all the National Research Council Guide for the Care 
and Use of Laboratory Animals guidelines. All experiments using mice were approved by the NIAID Animal Care and Use Committee. Mice were infected with approximately $100 \mathrm{CFU}$ of the H37Rv strain of Mtb using an aerosol chamber (Glas Col, Terre Haute, IN, USA).

To evaluate the effects of chemotherapy, mice infected for 28 days were treated with a cocktail containing the antibiotics rifampin (R) $(10 \mathrm{mg} / \mathrm{kg} / \mathrm{mouse})$, isoniazid $(\mathrm{H})(25 \mathrm{mg} / \mathrm{kg} /$ mouse), and pyrazinamide (Z) (150 mg/kg/mouse) (all from Sigma-Aldrich). After 60 days, treatment was changed to a cocktail consisting of $\mathrm{RH}$ alone. The drug stocks were prepared weekly and were orally administered by gavage 5 days per week. At the designated time points, mice were euthanized, blood was collected, and lungs were extracted and homogenized in PBS and stored at $-80^{\circ} \mathrm{C}$ for later analysis.

\section{Inbred and Mutant Mouse Strains Used As Source of Macrophages in In Vitro Experiments}

The donors were WT B6.SJL (CD45.1/1) mice or animals genetically deficient in the $\mathrm{HO}-1$ transcription factor NRF-2 (NRF2 $\left.{ }^{-/}\right)$, myeloid differentiation primary response gene $88\left(\mathrm{MyD}^{-/-}\right)$, TIR-domain-containing adapter-inducing interferon- $\beta$ $\left(\mathrm{TRIF}^{-/-}\right), \mathrm{IL}_{-10^{-/-}}, \mathrm{IFNAR}^{-/-}, \mathrm{FTH}^{-/-}$. Cells from $\mathrm{p} 47 \mathrm{phox}^{-/-}$or gp91 phox $^{-/-}$(two distinct subunits of the NADPH oxidase system) mice, which lack a fully functional NADPH-oxidase system, were also used. MyD88 ${ }^{-/-}$, $\mathrm{TRIF}^{-/-}$, IL10 ${ }^{-/-}$, and $\mathrm{IFNAR}^{-/-}$together with their respective WT controls were obtained from Taconic and $\mathrm{p}^{4} \mathrm{phox}^{-1-}$ (C57BL/6NTac background, Taconic) and gp91 hox $^{-1-}$ (C57BL/6J background, Jackson Lab) mice, were provided by Dr. Sharon Jackson (NIMHD, NIH). Bone marrow from $\mathrm{FTH}^{-/-}$and $\mathrm{NRF}^{-/-}$mice were provided by Dr. Miguel Soares (Instituto Gulbenkian de Ciências, Oeiras, Portugal). Bone marrow from miR-155KO mice were a gift from Dr. Stefan Muljo (NIAID, NIH). All mice were backcrossed for at least eight generations on a C57BL/6J or $\mathrm{N}$ background (indicated when exact genetic background was referred to original references or by investigator donating the animals for our use).

\section{Non-Human Primate Model of Pulmonary Mtb Infection}

The plasma samples analyzed were from animals utilized in a previously published study (37). In brief, 10 cynomolgus macaques (Macacca fascicularis) were infected with $M t b$ (Erdman strain) via bronchoscope, using an inoculum of 40-500 CFU (Table S1 in Supplementary Material). When the monkeys developed active TB (between 54 and 173 days postinfection; see Table S1 in Supplementary Material), they were treated daily with orally delivered isoniazid $(N=4)(15 \mathrm{mg} / \mathrm{kg} /$ dose $)$ or rifampin $(N=6)$ $(20 \mathrm{mg} / \mathrm{kg} /$ dose). Prior to treatment initiation and after 2 months of treatment, each macaque was given a PET/CT scan, using ${ }^{18} \mathrm{~F}-2$ fluoro-deoxyglucose (FDG) as a probe, and animals were matched for disease pattern in each treatment group. Change in FDG avidity in lungs over treatment was reported in Ref. (37). Plasma samples obtained from these animals were stored at $-80^{\circ} \mathrm{C}$ prior to analysis.

\section{In Vitro Experiments}

CD14 ${ }^{+}$column-purified human elutriated monocytes were obtained from peripheral blood of healthy donors from the $\mathrm{NIH}$ blood bank under Institutional Review Board-approved protocols of both the NIAID and the Department of Transfusion Medicine. Macrophages were generated by culturing monocytes in the presence of RPMI 1640 media containing 10\% human AB serum and M-CSF 50 ng/mL (PeproTech, Rocky Hill, NJ, USA) for 7 days; fresh media with growth factor were added every $48 \mathrm{~h}$, as previously described (71). This method of macrophage differentiation was chosen based on a recently published guideline (72). For infection experiments, cells were plated at a density of $10^{6}$ cells/well in 24 -well plates in phenol and serum-free media (Opti-MEM; Life Technologies, Carlsbad, CA, USA).

Murine BMDMs were employed in a second group of experiments. Bone marrow cells were cultured in 30\% L929 cellconditioned medium for 7 days. An additional $10 \mathrm{~mL}$ of L929 cell-conditioned medium were added after 4 days of incubation. The resulting adherent macrophages were detached with cold PBS and seeded in 96-well plates at $10^{5}$ cells/well, containing serum and phenol-free medium at $37^{\circ} \mathrm{C}$ in $5 \% \mathrm{CO}_{2}$.

Cells were exposed to WT H37Rv or an ESAT-6-deficient H37Rv mutant (a gift from Dr. Volker Briken, University of Maryland, College Park, MD, USA) Mtb strain at the indicated multiplicity of infection for $3 \mathrm{~h}$, washed to remove extracellular bacteria, and cultured in serum-free media for $24 \mathrm{~h}$ in the presence or absence of the indicated concentrations of Tempol (4-hydroxy-Tempo; 4-hydroxy-2,2,6,6-tetramethylpi-peridine$N$-oxyl, Sigma-Aldrich) or a TAT-conjugated NRF-2 peptide (TAT-14) that interacts with the Keap-1/NRF-2 complex (TOCRIS, Bristol, UK). In some experiments, recombinant ESAT-6 was delivered into the cytosol of infected macrophages using a fusion protein with the N-terminal fragment of the lethal factor of $B$. anthracis as previously described (10). The anthrax-protective Ag used in this procedure was also prepared as described previously (73). The two proteins at the indicated doses were added to cell cultures $3 \mathrm{~h}$ after $M t b$ infection and incubated for $24 \mathrm{~h}$. Cobalt (III) pro-toporphyrin IX dichloride (CoPPIX, Frontiers Scientific, Logan, UT, USA) was used as a positive control to induce HMOX1 mRNA expression in macrophages (Figure S2 in Supplementary Material). Culture supernatants were then collected, sterile filtered, and stored at $-80^{\circ} \mathrm{C}$ until use. Whole cell extracts were prepared following the manufacturer's instructions for the HO-1 ELISA kit (Enzo Life Sciences). Nuclear extracts were obtained using a kit from Active Motif (Carlsbad, CA, USA).

\section{Immunologic Measurement of Cellular Products}

Rabbit and mouse lungs were perfused with PBS and homogenized in PBS containing Complete Ultra protease inhibitor cocktail (Roche, Basel, Switzerland) and $2 \mathrm{mM}$ phenylmethylsulfonyl fluoride (Sigma-Aldrich). Rabbit HO-1 protein expression levels were quantified in plasma and lung homogenates (after normalization of their protein concentrations) using an ELISA kit (CUSABIO, College Park, MD, USA). HO-1 levels were measured in serum and lung homogenates of mice by ELISA (ADI-960-071, 
Enzo Life Sciences, Farmingdale, NY, USA) and in EDTA plasma samples from NHP using a human ELISA kit (ADI-EKS-800, Enzo Life Sciences). The same kit was used to measure HO-1 levels in plasma samples from TB patients as well as in whole cell extracts of the monocyte-derived macrophages studied in vitro.

Protein levels of the transcription factor NRF-2 in nuclear extracts from mouse macrophages were measured by ELISA (CUSABIO). NRF-2 activation was determined in nuclear extracts using TransAM DNA-binding ELISA kits (Active Motif, Carlsbad, CA, USA). Lipid peroxidation in culture supernatants was quantified using a kit (Cayman Chemical, Ann Harbor, MI, USA), which measures the formation of MDA. DNA/ RNA oxidation was quantitated in culture supernatants using an immunoassay from Cayman Chemical that detects all three oxidized guanine species: 8 -hydroxy-2'-deoxyguanosine from DNA, 8-hydroxyguanosine from RNA, and 8-hydroxyguanine from either DNA or RNA. Results from this assay were expressed as the concentration of $8-\mathrm{OH}-\mathrm{DG}$ in each sample. Intracellular production of ROS in macrophage cultures was assessed by staining cells with the oxidative fluorescent dye probe, dihydroethidium (DHE) $5 \mathrm{mM}$ (Invitrogen/Molecular Probes, Grand Island, NY, USA) for $30 \mathrm{~min}$ at $37^{\circ} \mathrm{C}$ in $5 \% \mathrm{CO}_{2}$, and then analyzed by flow cytometry, as described previously (11). The cells used for ROS assay had been previously detached from the culture plates using trypsin $(0.25 \%)$, washed, and then re-suspended in phenol and serum-free medium. Results were plotted as the mean fluorescence intensity of each of the experimental groups. Extracellular superoxide production was quantified as previously described (74) by adding hydroxylamine $(0.5 \mathrm{mM})$ during cell culture, which converts superoxide into nitrite plus nitrate, which were reduced by $\mathrm{VCl}_{3}$ treatment and quantified by Griess reaction (Sigma-Aldrich).

\section{HMOX1 Gene Expression Assay}

Total RNA was isolated from murine macrophages using the RNeasy Mini Kit, and residual DNA was digested using RNasefree DNase (both from QIAGEN, Valencia, CA, USA). The RNA samples were reverse transcribed using SuperScript II Reverse Transcriptase (Invitrogen, Carlsbad, CA, USA). Gene expression was measured using SYBR Green-based real-time quantitative PCR, and 18S mRNA was used as the housekeeping gene. The following oligonucleotide primers were used: $18 \mathrm{~S}$, forward, 5' -CACGGCCGGTACAGTGAAAC-3' and reverse, 5' -CCCGTC GGCATGTATTAGCT-3'; and HMOX1, forward, 5' -TCTCAGG GGGTCAGGTC-3' and reverse, 5'-GGAGCGGTGTCTGGGA TG-3'. Fold induction of HMOX1 gene expression was calculated using the DD threshold cycle method, normalizing mRNA levels for each sample to levels of $18 \mathrm{~S}$ and comparing with mRNA levels in unstimulated cells.

\section{Data Analyses}

The median values with interquartile ranges were used as measures of central tendency. For the in vitro experiments, bars represent mean and SD. The Mann-Whitney $U$ test (for two groups) or the Kruskal-Wallis test with the Dunn's multiple-comparison or non-parametric linear trend post hoc test (for more than two groups) were used to compare continuous variables in unmatched groups, whereas the Wilcoxon (for two groups) or Friedman (for more than two groups) matched-pairs tests were used in matched analysis, as indicated in table notes and figure legends. The Fisher's exact test or chi-square was used to compare variables displayed as percentages. Spearman rank tests were used to assess correlations. ROC curves were used to test the power of HO-1 levels to distinguish cure (successful treatment) from treatment failure or TB relapse at week 20 of ATT. All comparisons were prespecified and two-tailed. Differences with $p$ values below 0.05 after Holm's adjustment for multiple comparisons were considered statistically significant. The statistical analyses were performed using GraphPad Prism 7.0 (GraphPad Software, La Jolla, CA, USA), STATA 11 (StataCorp, College Station, TX, USA), JMP 13.0 (SAS, Cary, NC, USA), and R 3.1.0 (R Foundation, Vienna, Austria) programs.

\section{ETHICS STATEMENT}

The clinical study performed was approved by the Human Research Ethics Committee of the University of Cape Town, South Africa (approval number: 568/2012). Written informed consent was obtained from all study participants. The protocols, procedures, and animal care for the murine, rabbit, and non-human primate studies were approved, respectively, by the Institutional Animal Care and Use Committee from the National Institutes of Health (NIH), Bethesda, MD, USA (protocol number LPD99E); Johns Hopkins University, Baltimore, MD, USA (protocol number RB11M466); and University of Pittsburgh School of Medicine, Pittsburgh, PA, USA (protocol numbers A3187-01, 802011, and 808244). All the experiments were carried out in accordance with the recommendation of the Guide for the Care and Use of Laboratory Animals of the National Research Council of the National Academies of the United States of America (8th Edition) as mandated by the U.S. Public Health Service Policy. All animal experiments were performed in animal BSL-3 facilities maintained by the Intramural Research Program of National Institute of Allergy and Infectious Diseases (NIAID), Johns Hopkins University, and the University of Pittsburgh.

\section{AUTHOR CONTRIBUTIONS}

NR, DC, EA, BA, EB, AK, LG-S, KF, and CS performed experiments. NR, DC, EA, AS, RW, and BA designed experiments. AK, JF, SJ, KW, $\mathrm{WB}, \mathrm{AS}, \mathrm{RW}$, and BA provided materials and infrastructural support. DC, EA, AS, RW, and BA wrote the manuscript.

\section{ACKNOWLEDGMENTS}

The authors thank the study participants.

\section{FUNDING}

This project was supported in part by the Intramural Research Program of the NIAID and by a grant from the National Institutes of Health (NIH) U01AI115940. RW is supported by the Francis Crick Institute which receives its core funding from Cancer Research UK (FC00110218), the UK Medical Research Council 
(FC00110218), and the Wellcome Trust(FC00110218), Additional support was provided by the Wellcome Trust (104803), European Commission (Framework programme 7 research and innovation program under grant agreement FP7 HEALTH-F3-2012-305578 and Horizon 2020 research and innovation program under grant agreement No 643381), and National research Foundation of South Africa (96841). Non-human primate work was supported by grants to JF from the Bill and Melinda Gates Foundation (Drug Accelerator OPP42808 and Grand Challenges 11). The rabbit

\section{REFERENCES}

1. Houben RM, Dodd PJ. The global burden of latent tuberculosis infection: a re-estimation using mathematical modelling. PLoS Med (2016) 13(10): e1002152. doi:10.1371/journal.pmed.1002152

2. WHO. Global Tuberculosis Report 2015. Geneva, Switzerland: WHO Press (2015).

3. Mdluli K, Kaneko T, Upton A. The tuberculosis drug discovery and development pipeline and emerging drug targets. Cold Spring Harb Perspect Med (2015) 5(6):a021154. doi:10.1101/cshperspect.a021154

4. Orme IM, Robinson RT, Cooper AM. The balance between protective and pathogenic immune responses in the TB-infected lung. Nat Immunol (2015) 16(1):57-63. doi:10.1038/ni.3048

5. Salgame P, Geadas C, Collins L, Jones-Lopez E, Ellner JJ. Latent tuberculosis infection - revisiting and revising concepts. Tuberculosis (Edinb) (2015) 95(4): 373-84. doi:10.1016/j.tube.2015.04.003

6. Amaral EP, Lasunskaia EB, D'Imperio-Lima MR. Innate immunity in tuberculosis: how the sensing of mycobacteria and tissue damage modulates macrophage death. Microbes Infect (2016) 18(1):11-20. doi:10.1016/j.micinf. 2015.09.005

7. Ndlovu H, Marakalala MJ. Granulomas and inflammation: host-directed therapies for tuberculosis. Front Immunol (2016) 7:434. doi:10.3389/fimmu. 2016.00434

8. Palanisamy GS, Kirk NM, Ackart DF, Shanley CA, Orme IM, Basaraba RJ. Evidence for oxidative stress and defective antioxidant response in guinea pigs with tuberculosis. PLoS One (2011) 6(10):e26254. doi:10.1371/journal. pone. 0026254

9. Selek S, Aslan M, Horoz M, Celik H, Cosar N, Gunak F, et al. Peripheral DNA damage in active pulmonary tuberculosis. Environ Toxicol (2012) 27(6):380-4. doi:10.1002/tox.20674

10. Andrade BB, Pavan Kumar N, Amaral EP, Riteau N, Mayer-Barber KD, Tosh KW, et al. Heme oxygenase-1 regulation of matrix metalloproteinase-1 expression underlies distinct disease profiles in tuberculosis. JImmunol (2015) 195(6):2763-73. doi:10.4049/jimmunol.1500942

11. Amaral EP, Conceicao EL, Costa DL, Rocha MS, Marinho JM, CordeiroSantos M, et al. N-acetyl-cysteine exhibits potent anti-mycobacterial activity in addition to its known anti-oxidative functions. BMC Microbiol (2016) 16(1):251. doi:10.1186/s12866-016-0872-7

12. Lambeth JD. NOX enzymes and the biology of reactive oxygen. Nat Rev Immunol (2004) 4(3):181-9. doi:10.1038/nri1312

13. Liu XM, Peyton KJ, Ensenat D, Wang H, Schafer AI, Alam J, et al. Endoplasmic reticulum stress stimulates heme oxygenase-1 gene expression in vascular smooth muscle. Role in cell survival. J Biol Chem (2005) 280(2):872-7. doi:10.1074/jbc.M410413200

14. Gozzelino R, Jeney V, Soares MP. Mechanisms of cell protection by heme oxygenase-1. Апnu Rev Pharmacol Toxicol (2010) 50:323-54. doi:10.1146/ annurev.pharmtox.010909.105600

15. Prawan A, Kundu JK, Surh YJ. Molecular basis of heme oxygenase-1 induction: implications for chemoprevention and chemoprotection. Antioxid Redox Signal (2005) 7(11-12):1688-703. doi:10.1089/ars.2005.7.1688

16. Menzel DB, Rasmussen RE, Lee E, Meacher DM, Said B, Hamadeh H, et al. Human lymphocyte heme oxygenase 1 as a response biomarker to inorganic arsenic. Biochem Biophys Res Commun (1998) 250(3):653-6. doi:10.1006/ bbrc. 1998.9363

17. Wang L, Weng CY, Wang YJ, Wu MJ. Lipoic acid ameliorates arsenic trioxideinduced HO-1 expression and oxidative stress in THP-1 monocytes and study was supported by the Howard Hughes Medical Institute (to WB) and the NIH (R01 AI 079590, R01 AI037856, and R01 AI036973 to WB).

\section{SUPPLEMENTARY MATERIAL}

The Supplementary Material for this article can be found online at http://journal.frontiersin.org/article/10.3389/fimmu.2017.00542/ full\#supplementary-material.

macrophages. Chem Biol Interact (2011) 190(2-3):129-38. doi:10.1016/j. cbi.2011.02.001

18. Neubauer JA, Sunderram J. Heme oxygenase-1 and chronic hypoxia. Respir Physiol Neurobiol (2012) 184(2):178-85. doi:10.1016/j.resp.2012.06.027

19. Chang SH, Barbosa-Tessmann I, Chen C, Kilberg MS, Agarwal A. Glucose deprivation induces heme oxygenase-1 gene expression by a pathway independent of the unfolded protein response. J Biol Chem (2002) 277(3):1933-40. doi:10.1074/jbc.M108921200

20. Tsuchihashi S, Zhai Y, Fondevila C, Busuttil RW, Kupiec-Weglinski JW. HO-1 upregulation suppresses type 1 IFN pathway in hepatic ischemia/reperfusion injury. Transplant Proc (2005) 37(4):1677-8. doi:10.1016/j.transproceed.2005. 03.080

21. Chen C, Wang Y, Zhang Z, Wang C, Peng M. Toll-like receptor 4 regulates heme oxygenase-1 expression after hemorrhagic shock induced acute lung injury in mice: requirement of p38 mitogen-activated protein kinase activation. Shock (2009) 31(5):486-92. doi:10.1097/SHK.0b013e318188f7e1

22. Chen C, Zhang F, Zhang Z, Peng M, Wang Y, Chen Y. TLR4 signalinginduced heme oxygenase upregulation in the acute lung injury: role in hemorrhagic shock and two-hit induced lung inflammation. Mol Biol Rep (2013) 40(2):1167-72. doi:10.1007/s11033-012-2158-y

23. Silva-Gomes S, Appelberg R, Larsen R, Soares MP, Gomes MS. Heme catabolism by heme oxygenase-1 confers host resistance to Mycobacterium infection. Infect Immun (2013) 81(7):2536-45. doi:10.1128/IAI.00251-13

24. Kovtunovych G, Eckhaus MA, Ghosh MC, Ollivierre-Wilson H, Rouault TA. Dysfunction of the heme recycling system in heme oxygenase 1-deficient mice: effects on macrophage viability and tissue iron distribution. Blood (2010) 116(26):6054-62. doi:10.1182/blood-2010-03-272138

25. Fraser ST, Midwinter RG, Coupland LA, Kong S, Berger BS, Yeo JH, et al. Heme oxygenase-1 deficiency alters erythroblastic island formation, steadystate erythropoiesis and red blood cell lifespan in mice. Haematologica (2015) 100(5):601-10. doi:10.3324/haematol.2014.116368

26. Wallis RS, Doherty TM, Onyebujoh P, Vahedi M, Laang H, Olesen O, et al. Biomarkers for tuberculosis disease activity, cure, and relapse. Lancet Infect Dis (2009) 9(3):162-72. doi:10.1016/S1473-3099(09)70042-8

27. Andrade BB, Pavan Kumar N, Mayer-Barber KD, Barber DL, Sridhar R, Rekha VV, et al. Plasma heme oxygenase-1 levels distinguish latent or successfully treated human tuberculosis from active disease. PLoS One (2013) 8(5):e62618. doi:10.1371/journal.pone.0062618

28. Pavan Kumar N, Anuradha R, Andrade BB, Suresh N, Ganesh R, Shankar J, et al. Circulating biomarkers of pulmonary and extrapulmonary tuberculosis in children. Clin Vaccine Immunol (2013) 20(5):704-11. doi:10.1128/CVI.00038-13

29. Scharn CR, Collins AC, Nair VR, Stamm CE, Marciano DK, Graviss EA, et al. Heme oxygenase-1 regulates inflammation and mycobacterial survival in human macrophages during Mycobacterium tuberculosis infection. J Immunol (2016) 196(11):4641-9. doi:10.4049/jimmunol.1500434

30. Costa DL, Namasivayam S, Amaral EP, Arora K, Chao A, Mittereder LR, et al. Pharmacological inhibition of host heme oxygenase-1 suppresses Mycobacterium tuberculosis infection in vivo by a mechanism dependent on T lymphocytes. MBio (2016) 7(5):e01675-16. doi:10.1128/mBio.01675-16

31. Kumar A, Deshane JS, Crossman DK, Bolisetty S, Yan BS, Kramnik I, et al. Heme oxygenase-1-derived carbon monoxide induces the Mycobacterium tuberculosis dormancy regulon. J Biol Chem (2008) 283(26):18032-9. doi:10.1074/ jbc.M802274200

32. Shiloh MU, Manzanillo P, Cox JS. Mycobacterium tuberculosis senses hostderived carbon monoxide during macrophage infection. Cell Host Microbe (2008) 3(5):323-30. doi:10.1016/j.chom.2008.03.007 
33. Kubler A, Luna B, Larsson C, Ammerman NC, Andrade BB, Orandle M, et al. Mycobacterium tuberculosis dysregulates MMP/TIMP balance to drive rapid cavitation and unrestrained bacterial proliferation. J Pathol (2015) 235(3):431-44. doi:10.1002/path.4432

34. Kubler A, Larsson C, Luna B, Andrade BB, Amaral EP, Urbanowski M, et al. Cathepsin $\mathrm{K}$ contributes to cavitation and collagen turnover in pulmonary tuberculosis. J Infect Dis (2016) 213(4):618-27. doi:10.1093/infdis/jiv458

35. Mayer-Barber KD, Andrade BB, Oland SD, Amaral EP, Barber DL, Gonzales J, et al. Host-directed therapy of tuberculosis based on interleukin-1 and type I interferon crosstalk. Nature (2014) 511(7507):99-103. doi:10.1038/ nature 13489

36. Flynn JL, Capuano SV, Croix D, Pawar S, Myers A, Zinovik A, et al. Nonhuman primates: a model for tuberculosis research. Tuberculosis (Edinb) (2003) 83(1-3):116-8. doi:10.1016/S1472-9792(02)00059-8

37. Lin PL, Coleman T, Carney JP, Lopresti BJ, Tomko J, Fillmore D, et al. Radiologic responses in cynomolgous macaques for assessing tuberculosis chemotherapy regimens. Antimicrob Agents Chemother (2013) 57:4237-44. doi:10.1128/AAC.00277-13

38. Dutra FF, Bozza MT. Heme on innate immunity and inflammation. Front Pharmacol (2014) 5:115. doi:10.3389/fphar.2014.00115

39. Lee TS, Chau LY. Heme oxygenase-1 mediates the anti-inflammatory effect of interleukin-10 in mice. Nat Med (2002) 8(3):240-6. doi:10.1038/nm0302-240

40. Tzima S, Victoratos P, Kranidioti K, Alexiou M, Kollias G. Myeloid heme oxygenase-1 regulates innate immunity and autoimmunity by modulating IFN-beta production. J Exp Med (2009) 206(5):1167-79. doi:10.1084/jem. 20081582

41. Alam J, Stewart D, Touchard C, Boinapally S, Choi AM, Cook JL. Nrf2, a Capn'Collar transcription factor, regulates induction of the heme oxygenase-1 gene. J Biol Chem (1999) 274(37):26071-8. doi:10.1074/jbc.274.37.26071

42. Suzuki T, Yamamoto M. Molecular basis of the Keap1-Nrf2 system. Free Radic Biol Med (2015) 88(Pt B):93-100. doi:10.1016/j.freeradbiomed.2015. 06.006

43. Steel R, Cowan J, Payerne E, O'Connell MA, Searcey M. Anti-inflammatory effect of a cell-penetrating peptide targeting the Nrf2/Keap1 interaction. ACS Med Chem Lett (2012) 3(5):407-10. doi:10.1021/ml300041g

44. Lambeth JD, Neish AS. Nox enzymes and new thinking on reactive oxygen: a double-edged sword revisited. Annu Rev Pathol (2014) 9:119-45. doi:10.1146/ annurev-pathol-012513-104651

45. Jackson SH, Gallin JI, Holland SM. The p47phox mouse knock-out model of chronic granulomatous disease. J Exp Med (1995) 182(3):751-8. doi:10.1084/ jem.182.3.751

46. Pollock JD, Williams DA, Gifford MA, Li LL, Du X, Fisherman J, et al. Mouse model of X-linked chronic granulomatous disease, an inherited defect in phagocyte superoxide production. Nat Genet (1995) 9(2):202-9. doi:10.1038/ ng0295-202

47. Ronchi JA, Figueira TR, Ravagnani FG, Oliveira HC, Vercesi AE, Castilho RF. A spontaneous mutation in the nicotinamide nucleotide transhydrogenase gene of C57BL/6J mice results in mitochondrial redox abnormalities. Free Radic Biol Med (2013) 63:446-56. doi:10.1016/j.freeradbiomed. 2013.05.049

48. Brodin P, Rosenkrands I, Andersen P, Cole ST, Brosch R. ESAT-6 proteins: protective antigens and virulence factors? Trends Microbiol (2004) 12(11):500-8. doi:10.1016/j.tim.2004.09.007

49. Chandra S, Kaur M, Midha S, Bhatnagar R, Banerjee-Bhatnagar N. Evaluation of the ability of N-terminal fragment of lethal factor of Bacillus anthracis for delivery of Mycobacterium T cell antigen ESAT-6 into cytosol of antigen presenting cells to elicit effective cytotoxic T lymphocyte response. Biochem Biophys Res Commun (2006) 351(3):702-7. doi:10.1016/j.bbrc.2006.10.099

50. Kumar R, Halder P, Sahu SK, Kumar M, Kumari M, Jana K, et al. Identification of a novel role of ESAT-6-dependent miR-155 induction during infection of macrophages with Mycobacterium tuberculosis. Cell Microbiol (2012) 14(10):1620-31. doi:10.1111/j.1462-5822.2012.01827.x

51. Pamplona A, Ferreira A, Balla J, Jeney V, Balla G, Epiphanio S, et al. Heme oxygenase- 1 and carbon monoxide suppress the pathogenesis of experimental cerebral malaria. Nat Med (2007) 13(6):703-10. doi:10.1038/nm1586

52. Soares MP, Bach FH. Heme oxygenase-1: from biology to therapeutic potential. Trends Mol Med (2009) 15(2):50-8. doi:10.1016/j.molmed.2008.12.004
53. Luz NF, Andrade BB, Feijo DF, Araujo-Santos T, Carvalho GQ, Andrade D, et al. Heme oxygenase-1 promotes the persistence of Leishmania chagasi infection. J Immunol (2012) 188(9):4460-7. doi:10.4049/jimmunol.1103072

54. Paiva CN, Feijo DF, Dutra FF, Carneiro VC, Freitas GB, Alves LS, et al. Oxidative stress fuels Trypanosoma cruzi infection in mice. J Clin Invest (2012) 122(7):2531-42. doi:10.1172/JCI58525

55. Abdalla MY, Ahmad IM, Switzer B, Britigan BE. Induction of heme oxygenase-1 contributes to survival of Mycobacterium abscessus in human macrophages-like THP-1 cells. Redox Biol (2015) 4:328-39. doi:10.1016/j. redox.2015.01.012

56. Mitterstiller AM, Haschka D, Dichtl S, Nairz M, Demetz E, Talasz H, et al. Heme oxygenase 1 controls early innate immune response of macrophages to Salmonella typhimurium infection. Cell Microbiol (2016) 18:1374-89. doi: $10.1111 / \mathrm{cmi} .12578$

57. Stolt C, Schmidt IH, Sayfart Y, Steinmetz I, Bast A. Heme oxygenase-1 and carbon monoxide promote Burkholderia pseudomallei infection. J Immunol (2016) 197(3):834-46. doi:10.4049/jimmunol.1403104

58. Gill AJ, Kovacsics CE, Cross SA, Vance PJ, Kolson LL, Jordan-Sciutto KL, et al. Heme oxygenase-1 deficiency accompanies neuropathogenesis of HIVassociated neurocognitive disorders. J Clin Invest (2014) 124(10):4459-72. doi:10.1172/JCI72279

59. Gill AJ, Kovacsics CE, Vance PJ, Collman RG, Kolson DL. Induction of heme oxygenase-1 deficiency and associated glutamate-mediated neurotoxicity is a highly conserved HIV phenotype of chronic macrophage infection that is resistant to antiretroviral therapy. J Virol (2015) 89(20):10656-67. doi:10.1128/ JVI.01495-15

60. Diedrich CR, Flynn JL. HIV-1/Mycobacterium tuberculosis coinfection immunology: how does HIV-1 exacerbate tuberculosis? Infect Immun (2011) 79(4):1407-17. doi:10.1128/IAI.01126-10

61. Diedrich CR, O'Hern J, Wilkinson RJ. HIV-1 and the Mycobacterium tuberculosis granuloma: a systematic review and meta-analysis. Tuberculosis (Edinb) (2016) 98:62-76. doi:10.1016/j.tube.2016.02.010

62. Doitsh G, Galloway NL, Geng X, Yang Z, Monroe KM, Zepeda O, et al. Cell death by pyroptosis drives CD4 T-cell depletion in HIV-1 infection. Nature (2014) 505(7484):509-14. doi:10.1038/nature12940

63. Yang S, Li F, Jia S, Zhang K, Jiang W, Shang Y, et al. Early secreted antigen ESAT-6 of Mycobacterium tuberculosis promotes apoptosis of macrophages via targeting the microRNA155-SOCS1 interaction. Cell Physiol Biochem (2015) 35(4):1276-88. doi:10.1159/000373950

64. Keyse SM, Tyrrell RM. Heme oxygenase is the major $32-\mathrm{kDa}$ stress protein induced in human skin fibroblasts by UVA radiation, hydrogen peroxide, and sodium arsenite. Proc Natl Acad Sci U S A (1989) 86(1):99-103. doi:10.1073/ pnas.86.1.99

65. Applegate LA, Luscher P, Tyrrell RM. Induction of heme oxygenase: a general response to oxidant stress in cultured mammalian cells. Cancer Res (1991) 51(3):974-8.

66. Basu-Modak S, Tyrrell RM. Singlet oxygen: a primary effector in the ultraviolet A/near-visible light induction of the human heme oxygenase gene. Cancer Res (1993) 53(19):4505-10.

67. Kumar A, Farhana A, Guidry L, Saini V, Hondalus M, Steyn AJ. Redox homeostasis in mycobacteria: the key to tuberculosis control? Expert Rev Mol Med (2011) 13:e39. doi:10.1017/S1462399411002079

68. Tong KI, Kobayashi A, Katsuoka F, Yamamoto M. Two-site substrate recognition model for the Keap1-Nrf2 system: a hinge and latch mechanism. Biol Chem (2006) 387(10-11):1311-20. doi:10.1515/BC.2006.164

69. Maines MD, Kappas A. Enzymatic oxidation of cobalt protoporphyrin IX: observations on the mechanism of heme oxygenase action. Biochemistry (1977) 16(3):419-23. doi:10.1021/bi00622a012

70. WHO. Guidelines for Treatment of Tuberculosis. Geneva, Switzerland: WHO Press (2010)

71. Mayer-Barber KD, Andrade BB, Barber DL, Hieny S, Feng CG, Caspar P, et al. Innate and adaptive interferons suppress IL-1alpha and IL-1beta production by distinct pulmonary myeloid subsets during Mycobacterium tuberculosis infection. Immunity (2011) 35(6):1023-34. doi:10.1016/j. immuni.2011.12.002

72. Murray PJ, Allen JE, Biswas SK, Fisher EA, Gilroy DW, Goerdt S, et al. Macrophage activation and polarization: nomenclature and 
experimental guidelines. Immunity (2014) 41(1):14-20. doi:10.1016/j. immuni.2014.06.008

73. Liu S, Leung HJ, Leppla SH. Characterization of the interaction between anthrax toxin and its cellular receptors. Cell Microbiol (2007) 9(4):977-87. doi:10.1111/j.1462-5822.2006.00845.x

74. Khouri R, Bafica A, Silva Mda P, Noronha A, Kolb JP, Wietzerbin J, et al. IFN-beta impairs superoxide-dependent parasite killing in human macrophages: evidence for a deleterious role of SOD1 in cutaneous leishmaniasis. J Immunol (2009) 182(4):2525-31. doi:10.4049/jimmunol.0802860

Disclaimer: The funders had no role in study design, data collection, and interpretation or the decision to submit the work for publication.
Conflict of Interest Statement: The authors declare that the research was conducted in the absence of any commercial or financial relationships that could be construed as a potential conflict of interest.

Copyright (c) 2017 Rockwood, Costa, Amaral, Du Bruyn, Kubler, Gil-Santana, Fukutani, Scanga, Flynn, Jackson, Wilkinson, Bishai, Sher, Wilkinson and Andrade. This is an open-access article distributed under the terms of the Creative Commons Attribution License (CC BY). The use, distribution or reproduction in other forums is permitted, provided the original author(s) or licensor are credited and that the original publication in this journal is cited, in accordance with accepted academic practice. No use, distribution or reproduction is permitted which does not comply with these terms. 